\title{
Towards a More Desirable Dry Powder Inhaler Formulation: Large Spray-Dried Mannitol Microspheres Outperform Small Microspheres
}

\author{
Waseem Kaialy • Tariq Hussain • Amjad Alhalaweh • Ali Nokhodchi
}

Received: 24 March 2013 / Accepted: 24 June 2013

(C) Springer Science+Business Media New York 2013
Conclusion Particle shape and solid-state form of mannitol could dominate over carrier size, bulk density, flowability and charge in terms of determining the aerosolization behaviour of AS formulated with mannitol carrier, at least within the experimental protocols applied in the present study.

KEY WORDS aerosolization · albuterol sulphate · electrostatic charge $\cdot$ physicochemical properties · spray dried mannitol

\section{INTRODUCTION}

Mannitol is an attractive carrier in DPI systems due to its remarkable merits. These include for instance that mannitol gives a high sweet aftertaste that could be used to monitor the dose taken by the patient (1) and does not have a reducing effect making it a potential carrier for the aerosol delivery of proteins (2) and drug particles that contain a primary amine group (e.g., budesonide and formoterol).

The influence of carrier physicochemical properties on drug dispersion from dry powder inhalers is not completely

Electronic supplementary material The online version of this article (doi: I 0. I 007/s I | 095-0 I3- | | 32-2) contains supplementary material, which is available to authorized users.

W. Kaialy A. Nokhodchi

Chemistry and Drug Delivery Group, Medway School of Pharmacy

University of Kent, ME4 4TB Kent, UK

e-mail: a.Nokhodchi@kent.ac.uk

W. Kaialy $(\bowtie)$

Pharmaceutics and Pharmaceutical Technology Department

School of Pharmacy, University of Damascus, Damascus, Syria

e-mail: waseemkaialy@hotmail.co.uk

T. Hussain

The Wolfson Centre for Bulk Solids Handling Technology

Medway School of Engineering

University of Greenwich, Chatham Maritime, Kent, UK
A. Alhalaweh

ALS Scandinavia AB, Aurorum 10, 97775 Luleå, Sweden

A. Nokhodchi

Drug Applied Research Center and Faculty of Pharmacy

Tabriz University of Medical Sciences, Tabriz, Iran 
understood. In the literature, controversial reports can be found on the effect of these properties on drug aerosolisation efficiency. For example, both high (3) and low (4) fine particle fractions (FPF) of drug were documented by decreasing particle size of carrier particles. It is believed that the degree by which carrier particle size affects DPI formulation performance is influenced by other factors such as surface roughness (5), surface energy (6), type of drug (7), type of inhaler (8), chemical nature of carrier (9) and amounts of fine additives (10). A direct proportional relationship was reported between specific surface area of carrier particles and FPF of drug (11), but the reverse trend was found too (12). Good aerosolisation performance was observed when the bulk and the tap densities of the carrier powder were low (13) or high (14). Formulations with good (3) and poor (10) flow properties produced a high FPF of drug upon aerosolization. Both negative (15) and positive (16) relationships were established between carrier surface energy and FPF of drug. The FPF of drug increased with the elongation ratio of carrier particles employed within the formulation (17).

Indeed, studying the effect of one (independent) physicochemical property on DPI performance is challenging because manipulating one property could alter the others. Therefore, most studies focused on one property and gave less attention to other properties that could have varied alongside the particular property being studied. For example, in comparison to large particles of the same material, particles with smaller size could have smoother surfaces (18), have reduced relative degree of crystallinity, have increased surface energy (19), show high water uptake (20), and could generate powders with different charge density (21), high cohesive forces and poor flow properties.

The apparent disagreement among different reports could be partly attributed to the heterogeneous nature of carriers inspected. Therefore, it was the aim of this study to systematically evaluate the influence of particle size of a promising carrier, spray dried mannitol, on the performance of a DPI in the case of 'homogeneous' spherical carrier particles having a similar morphology, a similar true density and a similar solidstate form. This research was performed with a view to increase the knowledge of understanding the influence of carrier physicochemical properties on the performance of DPIs.

\section{MATERIALS AND METHODS}

\section{Materials}

Spray dried mannitol (SDM, Mannogem EZ Spray Dried) was sourced SPI pharma, UK. Micronized albuterol sulphate (AS, $d_{10 \%}=0.5 \pm 0.0 \mu \mathrm{m}, d_{50 \%}=1.7 \pm 0.1 \mu \mathrm{m}, d_{90 \%}=3.1 \pm 0.3 \mu \mathrm{m}$ (22)) was purchased from LB Bohle, Germany. For comparison purpose, $\alpha$-mannitol (acetone crystallized mannitol, ACM, prepared as described by Kaialy et al. (17)), $\beta$-mannitol (commercial mannitol A, CM-A, Fisher Scientific, UK) and $\delta$-mannitol (commercial mannitol B, CM-B, Parteck ${ }^{\circledR}$ Delta M, Merck KGaA, Darmstadt, Germany) were used for solid-state analyses.

\section{Sieving}

SDM powder was sieved to collect mannitol particles in different size fractions. Two different types of sieving were employed: mechanical sieving (mechanical shaker, Endecotts Ltd, UK) and air jet sieving (Copley Scientific, Nottingham, UK) operating at a gas volume flow that generates a negative pressure of $4 \mathrm{Kpa}$ and rotating nozzle speed of $50 \mathrm{rpm}$. Mannitol bulk powder was poured above the 125- $\mu \mathrm{m}$ sieve that has been placed on top of different sieves (Retsch ${ }^{\circledR}$ Gmbh Test Sieve, Germany) with smaller aperture sizes above one another as follows: $90-\mu \mathrm{m}$, $63-\mu \mathrm{m}, 45-\mu \mathrm{m}, 20-\mu \mathrm{m}$ and a metal collection plate. The mechanical shaker was operated for $30 \mathrm{~min}$, after which the amount of powder remained on top of each different sieve was further subjected to air jet sieving for $15 \mathrm{~min}$. Following the sieving process, particles with different size fractions [90-125 $\mu \mathrm{m}$ (SDM-A), 63-90 $\mu \mathrm{m}$ (SDM-B), 45-63 $\mu \mathrm{m}$ (SDM-C) and 20$45 \mu \mathrm{m}(\mathrm{SDM}-\mathrm{D})$ were collected and kept in sealed glass vials until used. All the analyses described below were performed on the sieved samples.

\section{Particle Size Measurements}

Particle size analysis was conducted using a Sympatec (Clausthal-Zellerfeld, Germany) laser diffraction-particle size analyzer as described in detail elsewhere (23). Mean diameter (MD, based on volume) and theoretical specific surface area (based on volume, assuming an ideal spherical smooth surface of the particles) were calculated by the Sympatic software. Ten determinations were measured for each sample.

\section{BET Analyses}

BET surface area was measured by nitrogen adsorption using Micromeritics Gemini 6 (Norcross, USA) automated gas sorption system model. The determination of external surface area was estimated by using the standard $t$-plot calculations by using experimental points at a relative pressure of $\mathrm{P} / \mathrm{P} 0=0.1-0.5$. All measurements were done in triplicate. Surface roughness of SDM particles was estimated according to equation 1 (Eq. 1).

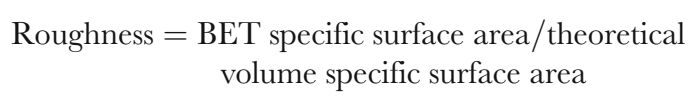

\section{Image Analysis Optical Light Microscopy}

Quantitative particle size and shape analysis were conducted using a computerized morphometric image analysis system 
(Leica DMLA Microscope; Leica Microsystems Wetzlar GmbH, Wetzlar, Germany; Leica Q Win Standard Analysing Software) (24). Particle size and geometric shape were quantified using several parameters including equivalent diameter $\left(D_{\text {equi }}\right)$, aspect ratio, convexity ratio, surface factor and sphericity (25). $\mathrm{D}_{\max }$ and $\mathrm{D}_{\min }$ were defined as the maximum and the minimum diameter of particle respectively.

\section{Scanning Electron Microscopy/Energy Dispersive $\mathrm{X}$-ray Analyses}

The use of only shape factors might not necessarily be sufficient to characterize particle shape. Therefore, all SDM particles were further quantitatively and qualitatively analyzed by scanning electron microscope (SEM). The powder samples were sputtered with chromium under argon vacuum using the sputter coater (Edwards S 150B Sputter Coater, $15 \mathrm{~nm}$ layer thickness). Electron micrographs with different magnifications of SDM samples were obtained using a SEM (HITACHI SU 8030, Japan) operating at $1-2 \mathrm{kV}$. The specimens were mounted on a metal stub with double-sided adhesive tape. Additionally, SEM images for AS powder and SDM:AS formulation blends were taken at different magnifications. Elemental composition energy dispersive $\mathrm{X}$-ray (EDX) chemical imaging analysis were carried out on albuterol sulphate-spray dried mannitol formulation samples using a Thermo-Noran system 7 detection system on the HITACHI SU 8030 FEG-SEM. A working distance of $8 \mathrm{~mm}$, an accelerating voltage of $5 \mathrm{kV}$ and a livetime of 100 s were used. Before analyses, samples were carbon coated to remove surface charging. Carbon coating was performed in the Polaron Emitech SC7640 Sputter Coater in a low-pressure atmosphere and a current of $100 \mathrm{~A}$ for $30 \mathrm{~s}$. Carbon coating was used because it does not disturb the $\mathrm{X}$-ray signals emitted from the sample and gives better signal strength.

\section{Water Content Analyses}

The moisture content of the SDM samples was determined semiautomatically by the Karl Fisher method (Metter Toledo, C20 Coulometric KF Titrator, Switzerland). The Fischer reagent solution was Hydranal ${ }^{\circledR}$ Coulomat AF (Sigma Aldrich, USA). The titration of each sample was repeated three times and an average value was taken.

\section{Differential Scanning Calorimetry}

A differential scanning calorimeter (DSC822, Mettler Toledo, Switzerland) was conducted as descried previously (14) to characterize the solid-state nature of different SDM samples. Each sample was heated from $25^{\circ} \mathrm{C}$ to $300^{\circ} \mathrm{C}$ at a heating rate of $30 \mathrm{~K} / \mathrm{min}$ (Such heating rate was shown previously to be preferable in terms of identification of $\delta$-mannitol from $\alpha$ - and $\beta$-mannitol (14)). In order to detect the presence of possible amorphous mannitol, all mannitol samples were analyzed by DSC from $-20^{\circ} \mathrm{C}$ to $250^{\circ} \mathrm{C}$ at a heating rate of $10 \mathrm{~K} / \mathrm{min}$. The melting enthalpies of all samples were calculated in triplicate by Mettler software.

\section{Fourier Transform Infrared Spectroscopy}

A SPECTRUM-ONE Fourier transform infrared (FT-IR) spectrometer (PerkinElmer, Massachusetts, USA) was used as descried previously (14) to analyse all SDM samples. A scanning range of $450-4,000 \mathrm{~cm}^{-1}$ with a $1 \mathrm{~cm}^{-1}$ resolution was used.

\section{Powder X-ray Diffraction}

The powder X-ray diffraction (PXRD) patterns of all SDM samples were collected on a Siemens DIFFRACplus 5,000 powder diffractometer with $\mathrm{Cu} \mathrm{K} \alpha$ radiation $(1.54056 \AA$ A according to the experimental procedure explained elsewhere (26). Quantitative mannitol crystal form analysis (\% $\quad \alpha-, \% \beta-$, or $\% \delta$-mannitol) were performed by Rietveld refinement using Topas v4 (Bruker) (26). The reproducibility of the PXRD patterns was verified by collecting multiple patterns of four different specimens of each sample. To obtain an accurate and quantitative phase analyses of different mannitol samples, all PXRD patterns were analyzed by the Rietveld refinement technique using TOPASAcademic V4 software. Lorentzian component convolutions of diffraction peaks varying in the diffraction angle $2 \theta$ as a function of $1 / \cos \theta$. Implemented into a whole pattern fit of the program TOPAS Academic V4, the approach yields domain sizes (crystallite sizes).

\section{Particle True Density Measurements}

True density of all SDM samples (particle mass/particle volume apart from both open and closed pores) was determined using an ultrapycnometer 1,000 (Quantachrom, USA) under helium gas (22). The mean of six determinations was recorded for each sample.

\section{Characterization of Bulk and Flow Properties of Spray Dried Mannitol Powders}

Bulk density (or apparent density), tap density (or drop density) and porosity (Eq. 2) of each mannitol powder sample were measured as important descriptors of powder bulk cohesive properties. Carr's index (CI, Eq. 3) was measured for all samples as an indication of powder flowability. The 
method incorporated was described elsewhere (27). Each sample was analysed five times.

$$
\begin{aligned}
& \text { Porosity }=\left(1-\frac{\text { Bulk density }}{\text { True density }}\right) \times 100 \\
& C I=\left(\frac{\text { Tap density }- \text { Bulk density }}{\text { Tap density }}\right) \times 100
\end{aligned}
$$

\section{Evaluation of Electrostatic Properties of Spray Dried Mannitol Powders}

A tribo-electric device electrostatic inductive sensor was used to investigate the tribo-electrification of SDM and AS bulk powders (28). Such novel method allows the detection and measurement of charge distribution on the charge sign basis in a population of particles. A sample of each powder was fed in the cylindrical sensor with the help of vibratory feeder and conveyed toward the sensor by gravity in a vertical direction. Special care was taken by considering the adhesion property of particles with the wall of the sensor. After each experiment, the inner tube was replaced in order to remove any deposits, impurities or surface charge that may have been present on the surface from a previous test. A fresh sample was used for each test experiment. Each sample was analyzed six times (humidity and temperature controlled laboratory: $50 \% \mathrm{RH}, 22^{\circ} \mathrm{C}$ ). The positive charge is the sum of positive charges whereas the negative charge is the sum of negative charges. The net charge is the sum of positive charges and negative charges, whereas the overall absolute charge is the sum of absolute charges detected regardless of sign of charge. The charge-to-mass ratio (CMR or charge density) was defined as the charge (negative charge for $\mathrm{N}-$ CMR, positive charge for $\mathrm{P}-\mathrm{CMR}$, net charge for netCMR, or overall absolute charge for absolute-CMR) per unit mass, in $\mathrm{nC} / \mathrm{g}$.

\section{Preparation of AS-Mannitol Formulations}

Each mannitol powder (5 g) was blended with AS powder (67.5: 1, w/w, Ventolin Rotacaps ${ }^{\circledR}$ ). This blending was performed in a cylindrical aluminium container $(6.5 \times 8 \mathrm{~cm})$ using a Turbula ${ }^{\circledR}$ mixer (Maschinenfabrik, Basel, Switzerland) at a constant speed of $100 \mathrm{rpm}$ for $30 \mathrm{~min}$ for the preparation of all formulations under similar protocol. Once prepared, all formulations were stored in tightly sealed glass vials for 7 days prior to any investigation until used.

\section{Quantification of Albuterol Sulphate}

Albuterol sulphate (AS) content was analysed using HPLC (Waters, USA). A mixture of methanol and $0.25 \%(w / v)$ 1-heptane sulfonic acid sodium salt $(45 / 55, v / v)$ was used as mobile phase. The flow rate of the mobile phase was $2 \mathrm{~mL} / \mathrm{min}$ and the assay wavelength was $200 \mathrm{~nm}$. The method incorporated was described in detail elsewhere (27).

\section{Drug Homogeneity Assessment}

After blending, ten randomly selected samples (33 $\pm 1.5 \mathrm{mg}$, equivalent to a unit dose of $\mathrm{AS}: 481 \pm 22 \mu \mathrm{g}$ ) were taken from different spots of each formulation powder for assay of AS content. Each sample was dissolved in $100 \mathrm{~mL}$ distilled water in a volumetric flask. For each formulation, \% potency was calculated as the percent amount of AS to the nominal dose. Per cent coefficient of variation $(\% \mathrm{CV})$ was calculated to determine the degree of AS content homogeneity.

\section{In Vitro Aerosolisation Study}

After blending, each formulation was filled manually into hard gelatin capsules (size 3 ) with $33 \pm 1.5 \mathrm{mg}$ powder. Before any investigation, all filled capsules were stored in sealed glass vials for at least $24 \mathrm{~h}$ in order to allow any charge-relaxation to occur. Pharmacopoeial deposition profiles of all formulations were assessed in vitro $\left(22^{\circ} \mathrm{C}, 50 \% \mathrm{RH}\right)$ using Aerolizer ${ }^{\circledR}$ inhaler device (Novartis, Switzerland) and a Multi-Stage Liquid Impinger (MSLI) equipped with a USP induction port (IP) (Copley Scientific, Nottingham, UK) at a flow rate that corresponds to a pressure drop of $4 \mathrm{kPa}$ across the device (92 L/min), as adopted from Kaialy et al. (23). Amounts of AS deposited on the inhaler with mouthpiece adaptor $(\mathrm{I}+\mathrm{M})$, induction port (IP, throat) and all stages of the MSLI were quantified. Ten capsules (ten doses) were actuated separately for each formulation tested, and the aerosolization test for each formulation was repeated four times (40 capsules in total). Several parameters were employed to quantify AS deposition profiles from each formulation including fine particle fraction $\left(\mathrm{FPF}_{\leq 5 \mu \mathrm{m}}\right)$, impaction loss (IL), dispersibility (DS), effective inhalation index (EI), mass median aerodynamic diameter (MMAD) and geometric standard deviation (GSD), as defined and explained previously (29).

\section{Overall Desirability}

Desirability function (DF) value is helpful in terms of evaluating several parameters concurrently and hence could be functional for experimental design-based optimization. To perform a quantitative comparison of different DPI formulations containing different SDM particles, a desirability function (di, Eq. 4) was defined (22):

$d_{i}=\frac{\Upsilon_{i}-\Upsilon_{\min }}{\Upsilon_{\max }-\Upsilon_{\min }}$ 
where $Y_{i}, Y_{\max }$ and $Y_{\min }$ are the actual observed, the maximum acceptable and the minimum acceptable values for the response variable respectively. Typically, optimum DPI formulation is required to demonstrate a good drug content homogeneity (low \% CV, important to attain uniform metering doses by the patient), good flow properties (low \% CI, essential to achieve good dose homogeneity, dose metering and industrial processing), minimal drug loss (low $\% \mathrm{I}+\mathrm{M})$, minimal deposition on throat (low \% IP) and maximal deposition on lower airways (high \% FPF, which could be, theoretically, directly related to biological effectiveness). Therefore, FPF, GV, CI and I+M+IP (\% deposition on $\mathrm{I}+\mathrm{M}+\mathrm{IP})$ indexes were selected as key parameters in comparative evaluation of different DPI formulations using overall desirability function (DF) as follows (Eq. 5, see Supplementary Material 1 for minimum and maximum response variables):

$$
D F=\left(d_{F P F}+\frac{1}{d_{C V}}+\frac{1}{d_{C I}}+\frac{1}{d_{I+M+I P}}\right)^{\frac{1}{4}}
$$

\section{RESULTS AND DISCUSSION}

\section{Particle Size Distribution for Spray Dried Mannitol}

SDM particles demonstrated considerably different particle size distributions (PSDs) as indicated by laser diffraction (volumeweight) (Fig. 1a, Supplementary Material 2) and further qualitatively and quantitatively (number-weight) ascertained by SEM (Fig. 2, Supplementary Material 3). All SDM particles exhibited symmetric, lognormal, mono-modal size distributions (Supplementary Material 2). Particle sizes at $10 \%\left(d_{10 \%}\right), 50 \%$ $\left(d_{50 \%}\right.$, median diameter $)$ and $90 \%\left(d_{90 \%}\right)$ volume distribution (Fig. la) and particle surface roughness (Fig. 1b) for different SDM samples were in the following rank order: SDM-A > SDM-B $>$ SDM-C $>$ SDM-D. On the other hand, the following rank order can be formulated for SDM particles in terms of both span (Fig. la) and BET surface area (Fig. 1b): SDM-A $<$ SDM-B $\approx$ SDM-C $<$ SDM-D. In general, a higher particle surface area could be related to smaller mean size, broader particle size distribution, higher surface energy (12) and/or higher specific charge (30).
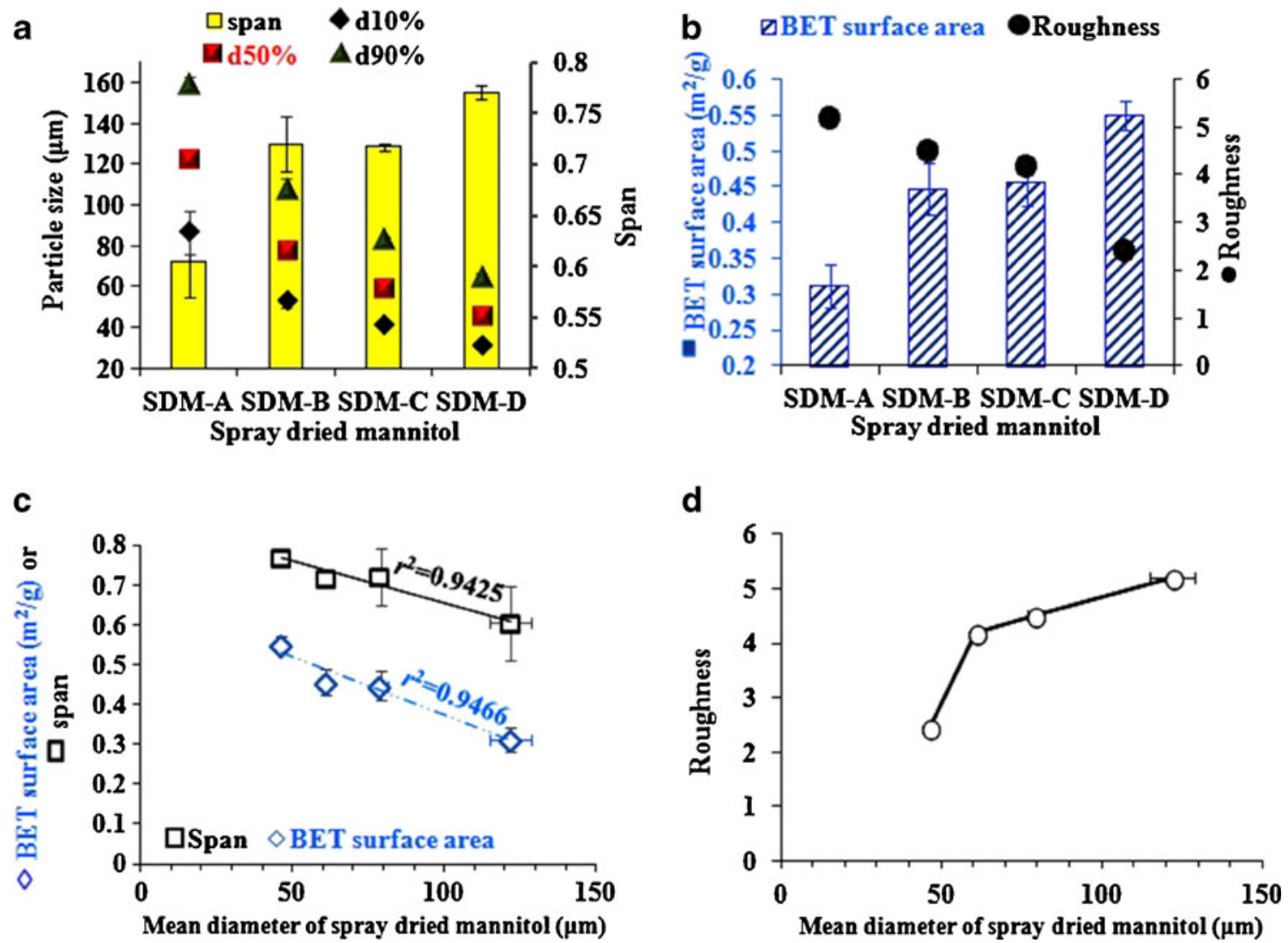

Fig. I Particle size at 10\% (black diamond suit, d10\%), 50\% (black square, d50\%, median diameter), 90\% (black up-pointning triangle, d90\%) volume distribution and span (a); BET surface area and roughness (black circle) (b) for spray dried mannitol (SDM) particles with different size fractions: $90-125 \mu \mathrm{m}$ (SDM-A), 63-90 $\mu \mathrm{m}$ (SDM-B), 45-63 $\mu \mathrm{m}$ (SDM-C) and 20-45 $\mu \mathrm{m}$ (SDM-D); mean diameter for different SDM particles (mean \pm SD, $n=10$ ) in relation to BET surface area (lozenge), span (white square) (c) and roughness of SDM particles (d). Smaller SDM particles have a higher specific surface area, a smoother surfaces, and generated relatively less-homogeneous powders. 
a

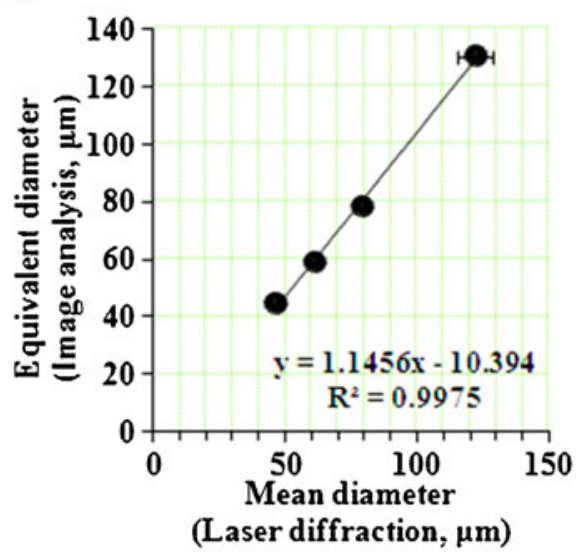

b
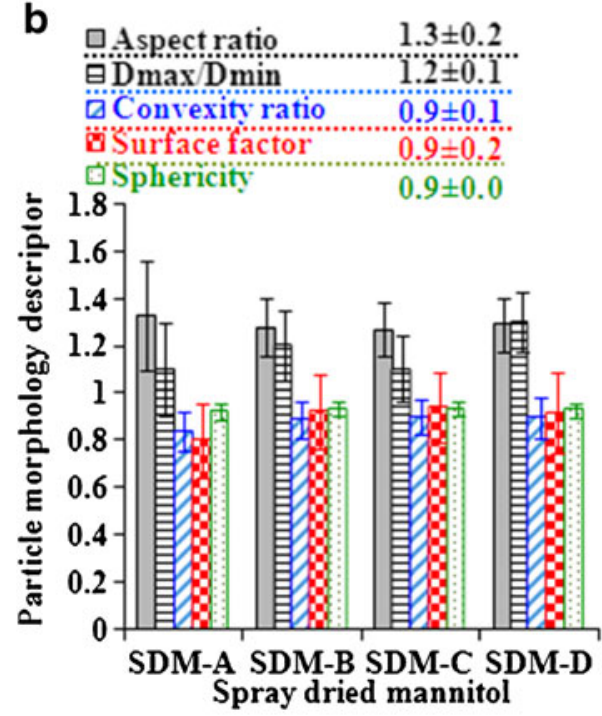

C

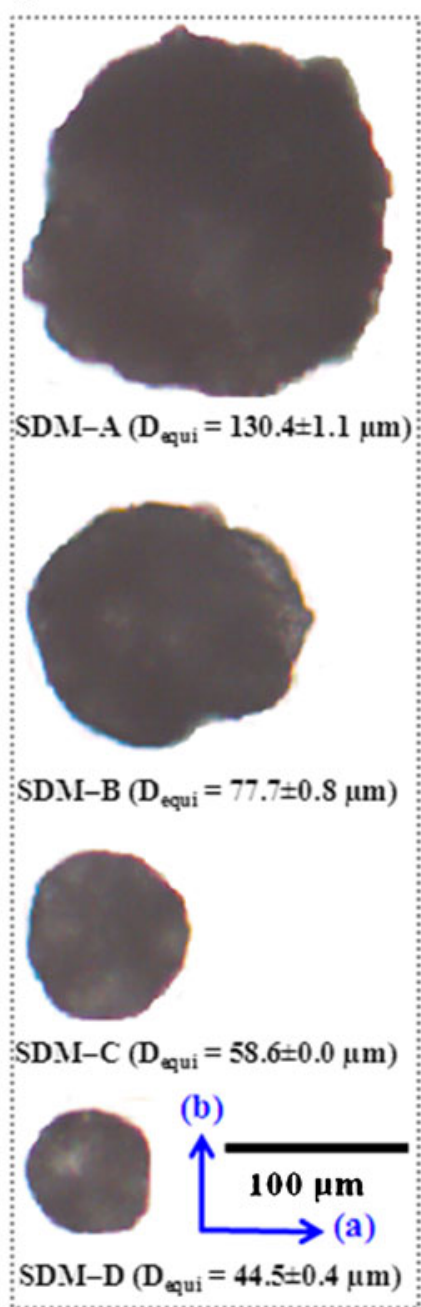

d
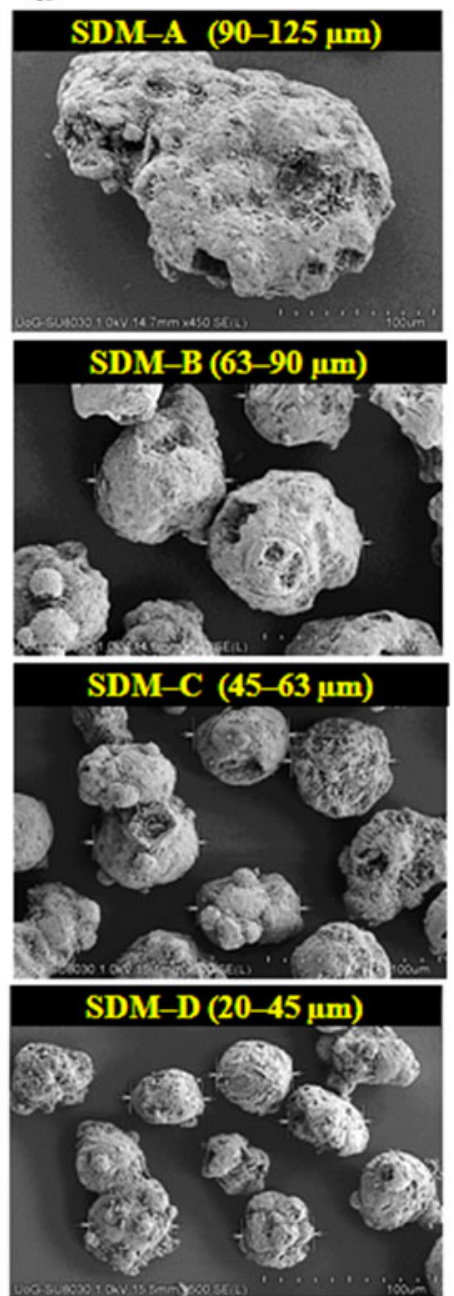

Fig. 2 Relationship between equivalent diameter ( $D_{\text {equi, }}$ mean $\pm S E, n \geq 1,000$ ) obtained by image analyses optical microscopy and mean diameter (mean $\pm S D, n=10)$ obtained by laser diffraction $(\mathbf{a})$; aspect ratio, $D_{\max } / D_{\min }$, convexity ratio, surface factor and sphericity $(\mathbf{b})$; optical microscope photographs $(\mathbf{c})$; and scanning electron photographs (d) for spray dried mannitol (SDM) particles with different size fractions: 90-125 $\mu$ m (SDM-A), 63-90 $\mu$ m (SDM-B), 45$63 \mu \mathrm{m}(\mathrm{SDM}-\mathrm{C})$ and 20-45 $\mu \mathrm{m}$ (SDM-D). SDM particles demonstrated a similar morphology.

Mostly, particle size of SDM samples fell into the nominal sieve size range, which could be due to uniform spherical shape of SDM particles (Figs. 1a and 2, Supplementary Material 2 and 3). Span value for all SDM powders ranged between $0.6 \pm 0.1$ and $0.8 \pm 0.0$ (Fig. 1a) indicating homogeneous size distributions ( $\operatorname{span}<1$ ). Such homogeneous size distribution is advantageous in terms of quality and effectiveness of the DPI formulation because high polydispersity of aerosol particle size might lead to high variations in lung deposition regions. Fine mannitol particles $(\leq 5 \mu \mathrm{m})$ were less than $1 \%(v / v)$ for all SDM samples (Supplementary Material 2), indicating that the applied sieving process was efficient to eliminate almost all fine particles. This is important in terms of direct comparison between different spherical SDM particles since fine carrier particles could considerably affect DPI performance (10).

Spherical SDM particles with smaller mean diameter (MD) demonstrated higher BET specific surface area (linear, $\left.r^{2}=0.9466\right)$ (Fig. 1c), smoother surfaces (Fig. 1d) and generated powders with relatively less homogeneous size distributions (higher span values, linear $r^{2}=0.9425$ ) (Fig. 1c).

\section{Particle Shape Analysis for Spray Dried Mannitol}

Particle size data provided by image analysis were in agreement with data provided by laser diffraction, since a direct linear relationship $\left(r^{2}=0.9975\right)$ was established between particle equivalent diameter $\left(D_{\text {equi }}\right.$, obtained by image analyses) and mean diameter (MD, obtained by laser diffraction) (Fig. 2a).

Statistically, all SDM particles demonstrated a similar $(P>0.05)$ aspect ratio $(1.3 \pm 0.2)$, a similar $\mathrm{D}_{\max } / \mathrm{D}_{\min }$ $(1.2 \pm 0.1)$, a similar convexity ratio $(0.9 \pm 0.1)$, a similar surface factor $(0.9 \pm 0.2)$ and a similar sphericity $(0.9 \pm 0.0)($ mean \pm SD, average of all samples) (Fig. 2b). This indicated that all SDM particles have a similar shape, which was further confirmed by 
the representative photographs of different SDM particles (Fig. 2c and d). All particle shape descriptors were around unity with a relatively small standard deviation $(\leq 0.2)$ indicating that all SDM particles were spherical-regular (uniform)shaped (Fig. 2b, c and d). Such similarity in terms of shape among different carrier particles is of importance in terms of straight evaluation between SDM particles because DPI performance is dependent on the particle shape of carrier $(4,17)$. In addition, shape-similarity and low degree of agglomeration could account for the agreement between image analysis and laser diffraction in terms of particle size determination of SDM particles (Fig. 2a-c). This is because both measurements obtained by laser diffraction and image analysis are more precise in the case of spherical particles.

\section{Water Content Analysis for Spray Dried Mannitol}

All SDM particles demonstrated a similar (negligible) amount of water content $(0.1 \pm 0.1 \%, w / w)$. This indicates the absence of mannitol hydrate form all SDM particles. Additionally, this indicates that, despite their relatively high surface area (Fig. 1b), smaller SDM particles did not show higher moisture content in comparison to larger particles. This could be attributed to the non-hygroscopic behaviour of mannitol (31), which is advantageous in terms of better physical and chemical stability of the DPI formulation, lower degree of solid bridging (by capillary forces which could cause irreversible powder aggregation) and better DPI formulation aerosolization (32). This suggests that SDM could be preferred in dry powder inhalation formulations compared to other sugars that have a hygroscopic nature such as xylitol and glucose.

\section{Solid-State Characterization of Spray Dried Mannitol Particles}

All DSC traces showed one endothermic transition at $169.9 \pm 0.1-171.3 \pm 1.6^{\circ} \mathrm{C}($ mean $\pm \mathrm{SD}, P>0.05)$ with similar melting enthalpy $(283.2 \pm 3.6-292.5 \pm 4.2 \mathrm{~J} / \mathrm{g}$, mean $\pm \mathrm{SD}$, $P>0.05$ ) (Fig. 3a). Such DSC curves suggest the presence of $\alpha-$ mannitol and/or $\beta$-mannitol crystal form. There was no detectable $\delta$-mannitol (which would have produced a melting endotherm at $150-158^{\circ} \mathrm{C}$ ) (14) or free water (or surface water, which would have produced an endothermic transition at around $100^{\circ} \mathrm{C}$ ) in the SDM samples. The absence of free water was also confirmed by Karl Fischer analysis. Additionally, the absence of any glass transition event around $13^{\circ} \mathrm{C}$ from the DSG traces (Supplementary Material 4) confirms that there was no detectable amount of amorphous mannitol within all mannitol samples. Similar melting enthalpy (Fig. 3a) suggests a similar relative degree of crystallinity for different SDM particles. In addition, SDM samples demonstrated similar melting enthalpy to the mannitol control samples (ACM, CM-A and CM-B $)(253.3 \pm 5.1-254.8 \pm 3.9$ versus $251.0 \pm 2.4-$
$253.2 \pm 4.1 \mathrm{~J} / \mathrm{g}$ ). This suggested that SDM particles have a similar degree of crystalline nature to the control mannitol samples (Supplementary Material 4).

Mannitol exists in three common polymorphic forms, i.e., $\alpha-, \beta$ - and $\delta$-. Since pure $\alpha-$ and $\beta$-mannitol are indistinguishable by DSC (23), all SDM particles were further analysed by FT-IR (Fig. 3b) and PXRD (Fig. 4). All samples exhibited similar FT-IR spectra indicating their similar molecular structure (Fig. 3b). The presence of sharp, characteristic diffraction angles with no halo, background or peak widening in the PXRD patterns of all samples confirms the crystalline nature of all SDM samples (Fig. 4). All SDM particles exhibited $\alpha-$ mannitol specific FT-IR band at $1,194 \mathrm{~cm}^{-1}, \beta$-mannitol

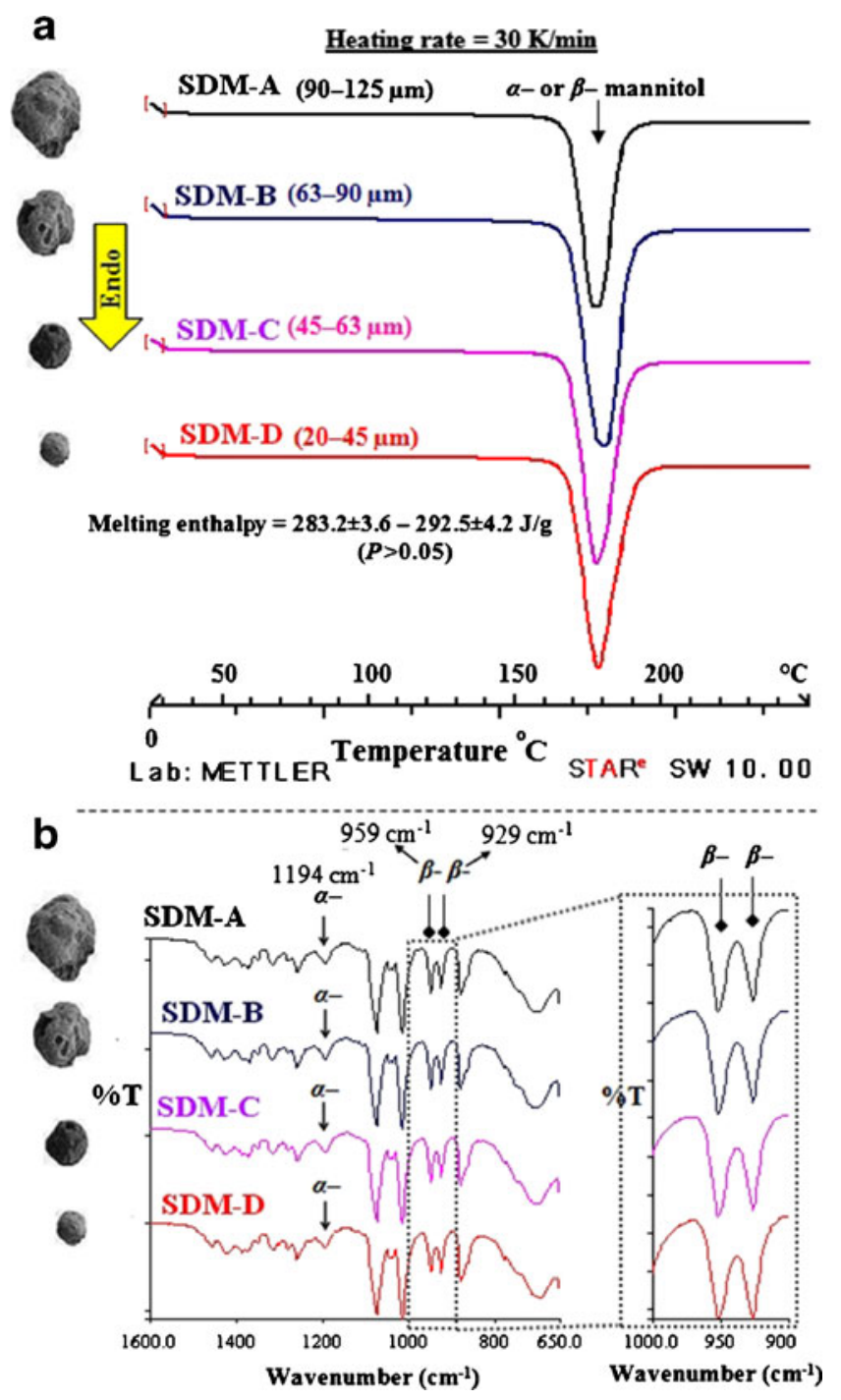

Fig. 3 Differential scanning calorimeter (DSC) traces heated from $25^{\circ} \mathrm{C}$ to $300^{\circ} \mathrm{C}$ at a heating rate of $30 \mathrm{~K} / \mathrm{min}(\mathbf{a})$; and fourier transform infrared (FTIR) spectra (from $650 \mathrm{~cm}^{-1}$ to I,600 $\mathrm{cm}^{-1}$ ) (b) for spray dried mannitol (SDM) particles with different size fractions: 90-125 $\mu \mathrm{m}$ (SDM-A), 63$90 \mu \mathrm{m}$ (SDM-B), 45-63 $\mu \mathrm{m}$ (SDM-C) and 20-45 $\mu \mathrm{m}$ (SDM-D) (\%T: percent transmittance). 
Fig. 4 PXRD patterns and \% solid-state polymorphic form (mean $\pm \mathrm{SD}, n=4$ ) for spray dried mannitol (SDM) particles with different size fractions: $90-125 \mu \mathrm{m}$ (SDM-A), 63-90 $\mu \mathrm{m}$ (SDM-B), 45-63 $\mu \mathrm{m}$ (SDM-C) and 20$45 \mu \mathrm{m}$ (SDM-D). SDM particles demonstrated a similar solid-state form (mixture of $\alpha$ - and $\beta$ mannitol).
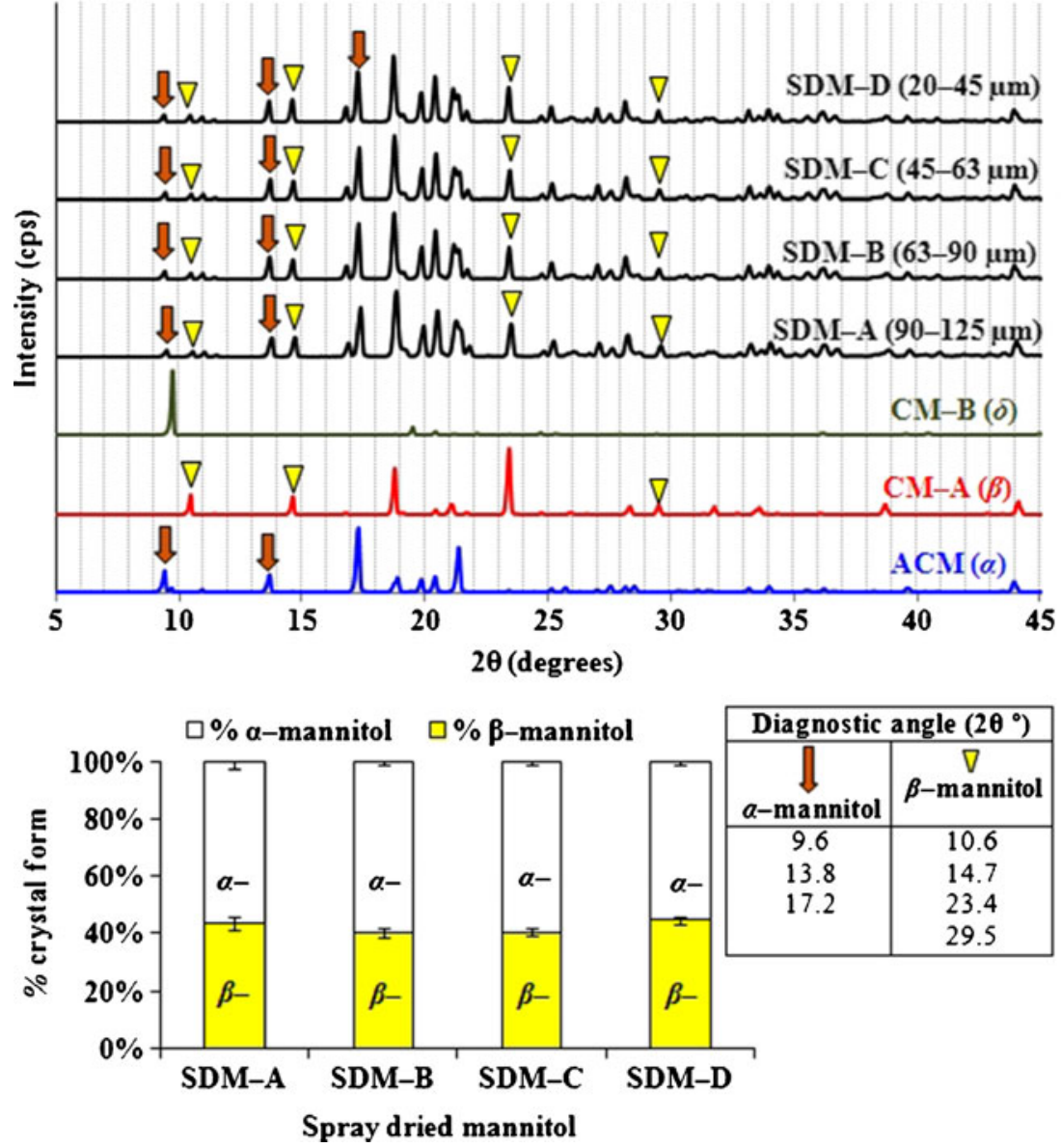

specific FT-IR bands at $959 \mathrm{~cm}^{-1}$ and $929 \mathrm{~cm}^{-1}$ (Fig. 3b), $\alpha-$ mannitol PXRD specific peaks at $2 \theta$ of $9.57,13.79$ and 17.2 and $\beta$-mannitol PXRD specific peaks at $2 \theta$ of $10.56,14.71$, 23.4 and 29.5 (Fig. 4). This indicates that all SDM samples consist of mixtures of $\alpha$-mannitol $(58 \pm 2 \%)$ and $\beta$-mannitol $(42 \pm 2 \%)$ with no significant $(P>0.05)$ difference between all samples (Fig. 4). The specific FT-IR band of $\delta$-mannitol (at $967 \mathrm{~cm}^{-1}$ ) and the PXRD diagnostic angle of $\delta$-mannitol (at $9.7^{\circ}$ and $22.2^{\circ}$ ) were absent from FT-IR spectra (Fig. 3b) and PXRD patterns (Fig. 4) of all SDM samples, which agrees with the DSC results (Fig. 3a). Structural PXRD data analyses showed that ACM $(\alpha$-mannitol), CM-A $(\beta$-mannitol) and CM-B $(\delta$-mannitol $)$ have volume-weighted mean crystallite sizes (LVol-IB) between 466 and $652 \mathrm{~nm}$ (Supplementary Material 5). SDM particles demonstrated a mean crystallite size between 517 and $930 \mathrm{~nm}$ (Supplementary Material 5). Such values confirm the practically absolute crystalline nature of all mannitol samples, which agrees with DSC results (Supplementary Material 4). Indeed, it is well established in the literature that spray dried mannitol could have a crystalline solid-state which is one of the advantages of using mannitol over lactose in DPI systems (33).

In conclusion, SDM particles with different size distributions demonstrated similar polymorphic form $(\alpha-+\beta-$ mannitol mixture), allowing the comparison of the influence of particle size of uniform spherical SDM particles on the DPI performance (6).

\section{Characterization of Density, Porosity and Flowability for Spray Dried Mannitol Powders}

All SDM particles demonstrated similar true density $\left(1.42 \pm 0.02-1.45 \pm 0.02 \mathrm{~g} / \mathrm{cm}^{3}, P>0.05\right)$ (Supplementary Material 6). This is beneficial for comparison between different mannitol particles because carrier particles with a lowdensity would have lower settling velocity, smaller aerodynamic diameter in comparison with their geometric diameter and a lower probability of impaction (due to lower inertial forces generated during aerosolization) (34).

SDM samples demonstrated considerably different bulk densities (from $0.42 \pm 0.01$ to $0.49 \pm 0.01 \mathrm{~g} / \mathrm{cm}^{3}$ ) and different tap densities (from $0.48 \pm 0.01$ to $0.58 \pm 0.02 \mathrm{~g} / \mathrm{cm}^{3}$ ), both were in the following rank order: SDM-A $<$ SDM-B $\leq$ SDM-C $<$ SDM-D (Supplementary Material 6). CI for different SDM powders was within the following rank order: SDM-A < SDM-B $<$ SDM-C $<$ SDM-D (Supplementary Material 6) ranging from $12.8 \pm 1.1 \%$ for $\mathrm{SDM}-\mathrm{A}$ (good flow character) to $21.8 \pm 1.1 \%$ for SDM-D (fair or reasonable flow character). 

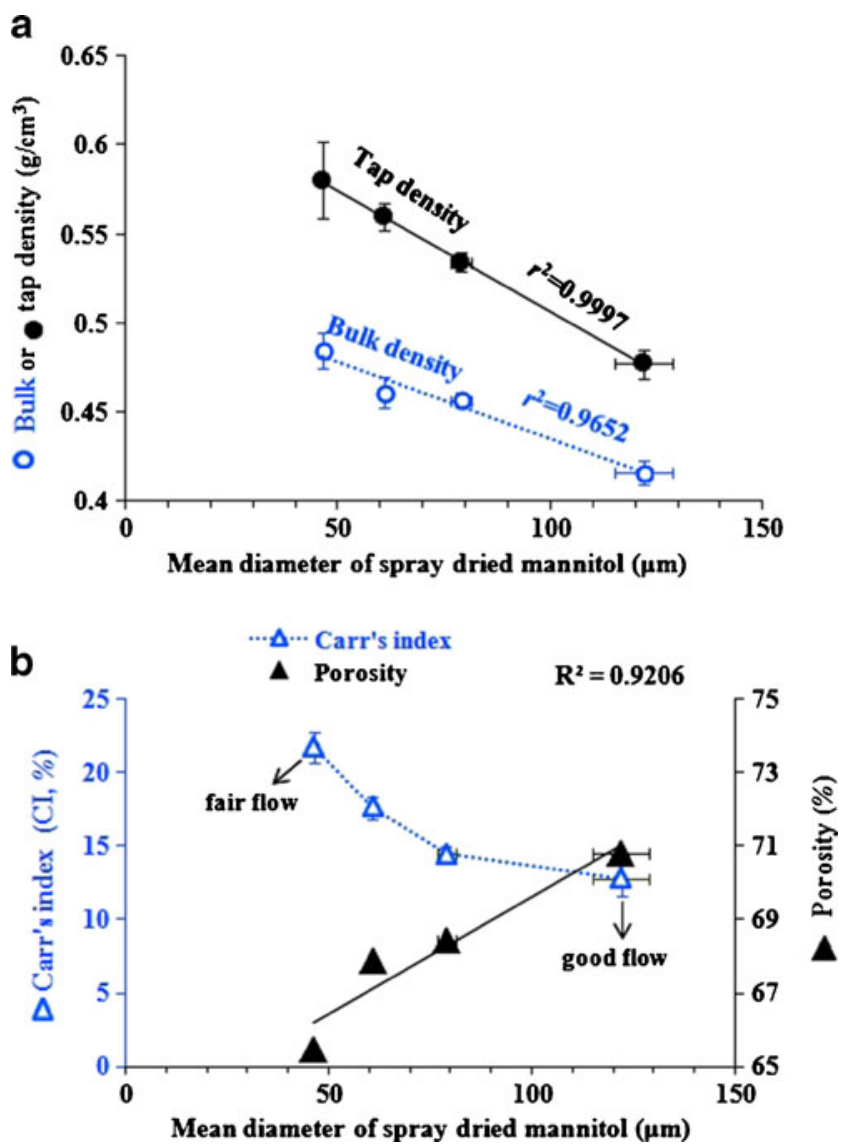

Fig. 5 Bulk density (white circle), tap density (black circle) (mean $\pm S D, n=5)(\mathbf{a})$; Carr's index $(\mathrm{Cl}$ ) (increment) and porosity (black up-pointing triangle) (mean $\pm \mathrm{SD}$, $n=5)(\mathbf{b})$ in relation to mean diameter (mean $\pm S D, n=10$ ) of spray dried mannitol (SDM) particles with different size fractions: 90-125 $\mu \mathrm{m}$ (SDM-A), 63-90 $\mu \mathrm{m}$ (SDM-B), 45-63 $\mu \mathrm{m}$ (SDM-C) and 20-45 $\mu \mathrm{m}$ (SDM-D). Bulk density and tap density increased with decreasing particle size of SDM.
Porosity for different SDM powders was in the following order: SDM-A $>$ SDM-B $>$ SDM-C $>$ SDM-D (Supplementary Material 6) ranging from 65.5 to $70.8 \%$. The results showed that the smaller the mean diameter of SDM particles the higher the bulk density (linear, $r^{2}=0.9652$ ), the higher the tap density (linear, $r^{2}=0.9997$ ) (Fig. 5a), the poorer the flowability (higher CI) and the smaller the porosity (linear, $r^{2}=0.9206$ ) (Fig. 5b) of SDM powder. This could be explained as the arrangement between small particles is more difficult than large particles.

Unlike bulk density and tap density, which are powder descriptors, true density is a particle feature. The results indicate that particle size had a negligible effect on the true density of SDM particles, which could be related to their similar solidstate form (Figs. 3 and 4). However, smaller SDM particles are more cohesive which could be due to the higher interparticulate average contact points between smaller particles.

\section{Electrostatic Charge of Spray Dried Mannitol and Albuterol Sulphate Powders}

The charge properties of SDM and AS powders (Fig. 6) were analysed using a recent novel approach developed in our laboratory (28). Each peak of the signals represents a charged particle travelling through the sensor. The directionality of each peak determines the charging sign whereas the height of the peak determines the level (magnitude) of charge (28). SDM particles with smaller size $(20-45 \mu \mathrm{m}$ and $45-63 \mu \mathrm{m})$ carried almost only electropositive charge behaviour (Fig. 6). Larger SDM particles (63-90 $\mu \mathrm{m}$ and 90-125 $\mu \mathrm{m}$ ) exhibited a bipolar charge trend. More particles were electropositively charged in the case of $63-90 \mu \mathrm{m}$ particles whereas more particles were
Fig. 6 Particle charge pattern (Hussain et al. (28)) for albuterol sulphate (all particles are electronegative) and spray dried mannitol (SDM) particles with different size fractions: $90-125 \mu \mathrm{m}$ (SDM-A, particles are electropositive and electronegative), 63-90 $\mu \mathrm{m}$ (SDM-B, particles are electropositive and electronegative), 45-63 $\mu \mathrm{m}$ (SDM-C, all particles are electropositive) and 20-45 $\mu \mathrm{m}$ (SDM-D, all particles are electropositive).

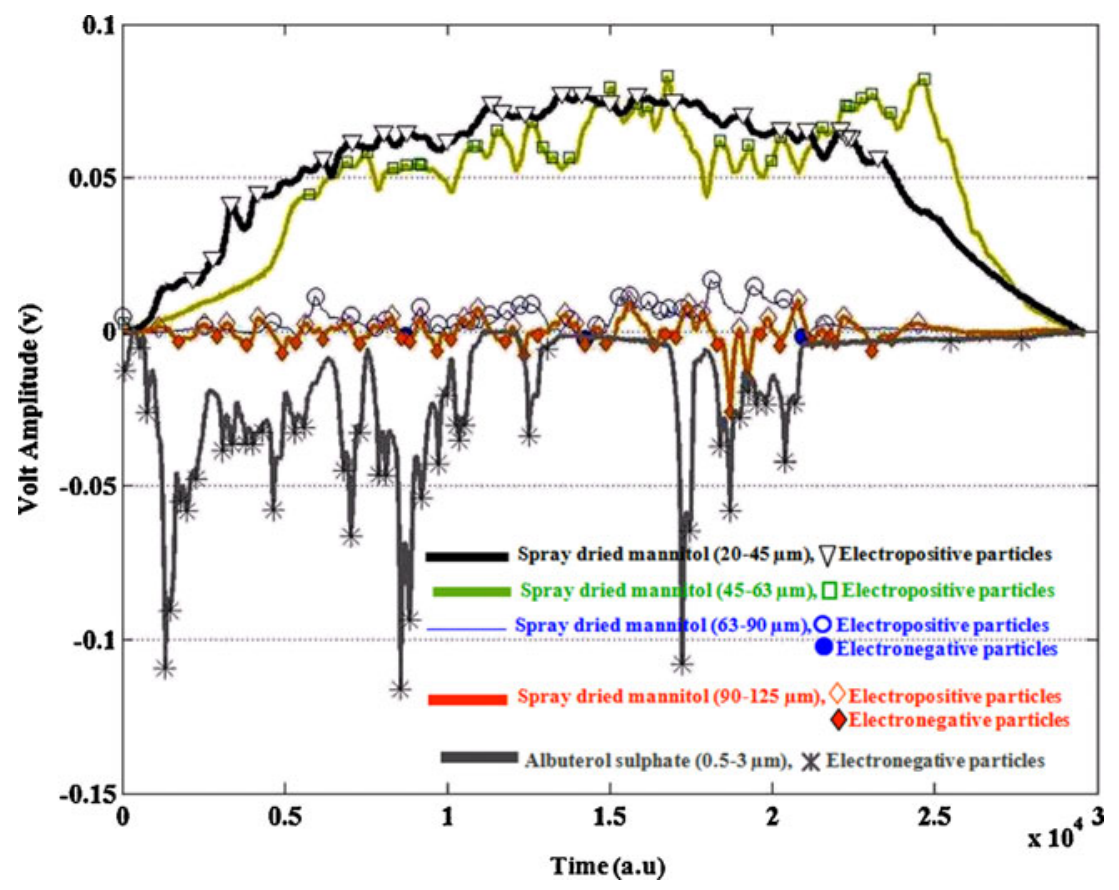


a
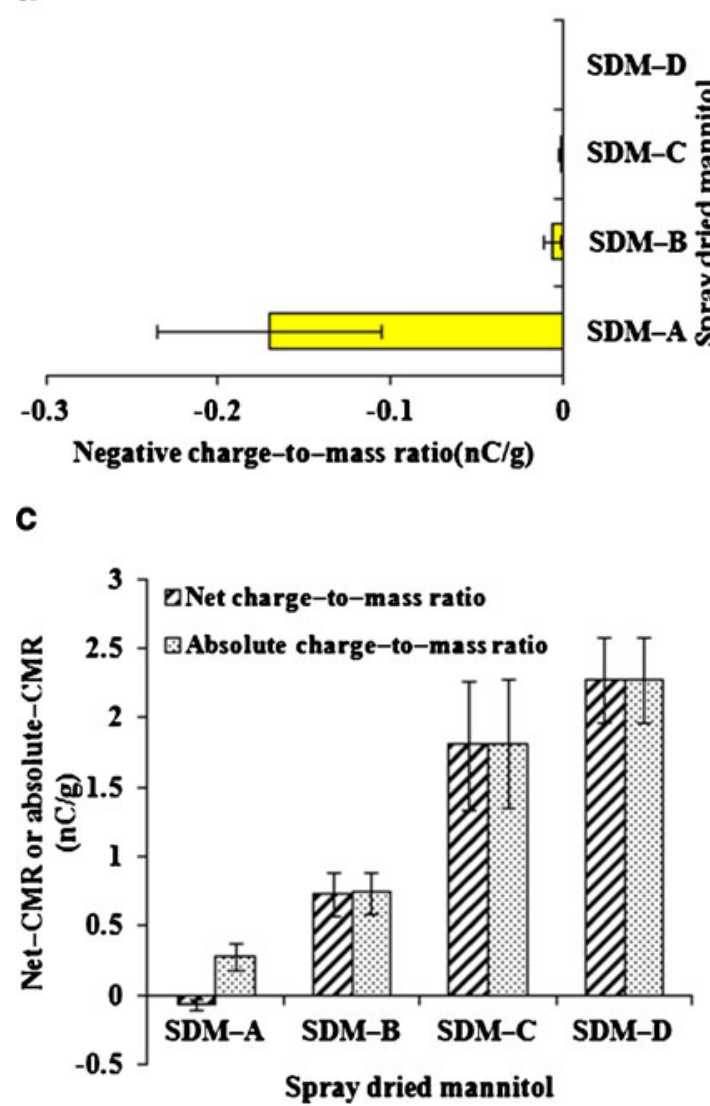

b

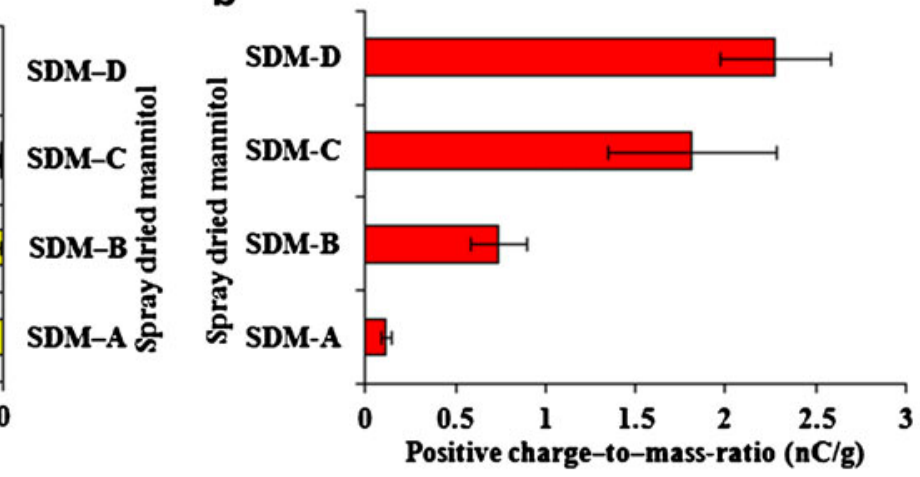

d

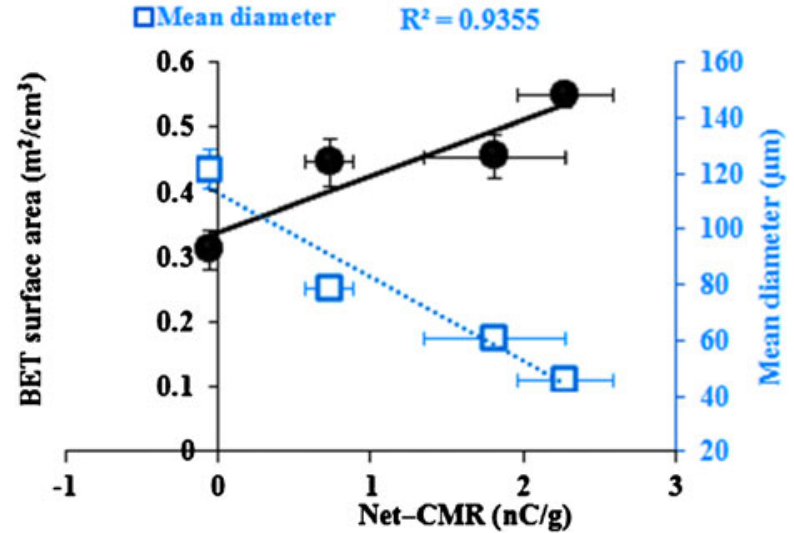

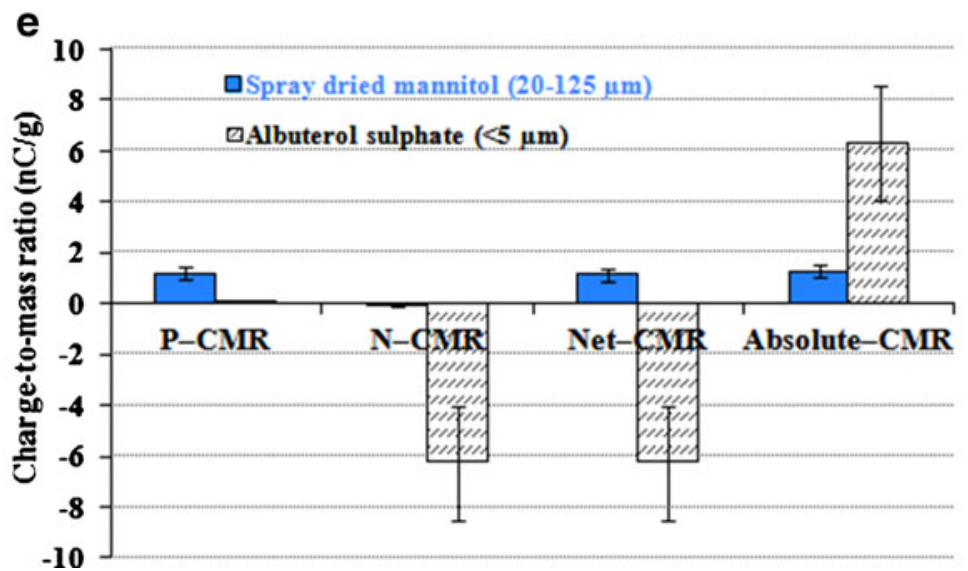

Fig. 7 Negative charge-to-mass ratio (N-CMR) (a), positive charge-to-mass ratio (P-CMR) (b), net charge-to-mass ratio (Net-CMR), absolute chargeto-mass ratio (absolute-CMR) (nano coulomb/gram: $\mathrm{nC} / \mathrm{g}$, mean $\pm \mathrm{SD}, n=6)(\mathbf{c})$ for spray dried mannitol (SDM) particles with different size fractions: 90

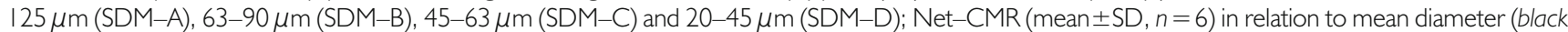
circle) (mean $\pm S D, n=10$ ) and BET specific surface area (O) (mean $\pm D, n=3$ ) of different SDM particles (d); P-CMR, N-CMR, net-CMR and absoluteCMR for spray dried mannitol (20-125 $\mu \mathrm{m}$, mean \pm SE, $n=24$ average of all samples) and albuterol sulphate $(<5 \mu \mathrm{m}$, mean \pm SE, $n \geq 6)(\mathbf{e})$. Charge density of SDM is higher for SDM particles with smaller size and higher surface area. Albuterol sulphate drug has a different charge sign and a higher charge density than spray dried mannitol carrier.

electronegatively charged in the case of $90-125 \mu \mathrm{m}$ sieved (Fig. 6). Unlike SDM powder, only strong electronegative charge behaviour was observed for AS powder (Fig. 6).

The quantitative charging trends of different SDM particles are shown in Fig. 7a (negative charge-to-mass ratio, $\mathrm{N}-\mathrm{CMR}$ ) and Fig. 7b (positive charge-to-mass ratio, $\mathrm{P}-\mathrm{CMR}$ ).
Bipolar charging was present in the case of SDM-A and SDM-B powders, whereas for SDM-C and SDM-D almost only an electropositive charge was detected (Figs. 7-a, -b). The smaller the size of the spherical SDM particles the smaller was the negative charge density (Fig. 7a) and the higher was the positive charge density (Fig. 7b). Indeed, the relationship 
between particle size and charge sign is a complex phenomenon because it is dependent on both material chemical structure and the nature of contact surfaces (35). Nevertheless, spherical mannitol particles with smaller size demonstrated higher net charge density and higher absolute charge density (Fig. 7c). This could be attributed to the relatively higher surface area/mass ratio (Fig. 1b), higher particle--wall contacts and greater particle number density in the case of smaller particles. Fig. 7d shows that the net charge density of mannitol powders increases with decreasing mean particle size and increasing surface area of the spherical mannitol particles.

In comparison to SDM, regardless of the size of SDM particles, albuterol sulphate (AS) particles demonstrated a negligible positive charge density, a significantly higher negative charge density, a higher net charge density with opposite sign of charge and a higher absolute charge density (Fig. 7e). This could be attributed to the relatively large size $(20-125 \mu \mathrm{m})$ and spherical morphology (Fig. 2) for SMD particles in comparison to AS particles $(<5 \mu \mathrm{m})$. Generally, spherical particles are expected to have less total charge than the angular (irregular) particles (e.g., AS, as shown later) since electrical charges are likely to distribute homogeneously on spherical particles. Also, mechanically micronized particles, such as albuterol sulphate, are usually prepared by collision between coarse particles which could generate a high electrical charge.

Individual charged drug particles could induce an opposite image charges in the upper airway regions accelerating drug deposition in this region. In addition, drug-carrier adhesion forces could be high in the case of drug particles and carrier particles with opposite charges (36), such as albuterol sulphate and mannitol investigated in this study (Fig. 7e). This could be disadvantageous because it is expected to discourage AS-mannitol detachment during aerosolization.

\section{DPI Formulation Assessments}

\section{Drug Content Homogeneity}

All formulations achieved uniform drug content with $\mathrm{CV}$ of less than $6 \%(2.0-5.4 \%$, Fig. 8a). This suggests that the overall procedures of sampling, dissolving and drug analysis were practically accurate and reproducible with satisfactory mixing. Acceptable drug content homogeneity could be attributed to the homogeneous size distributions (Fig. 1a),
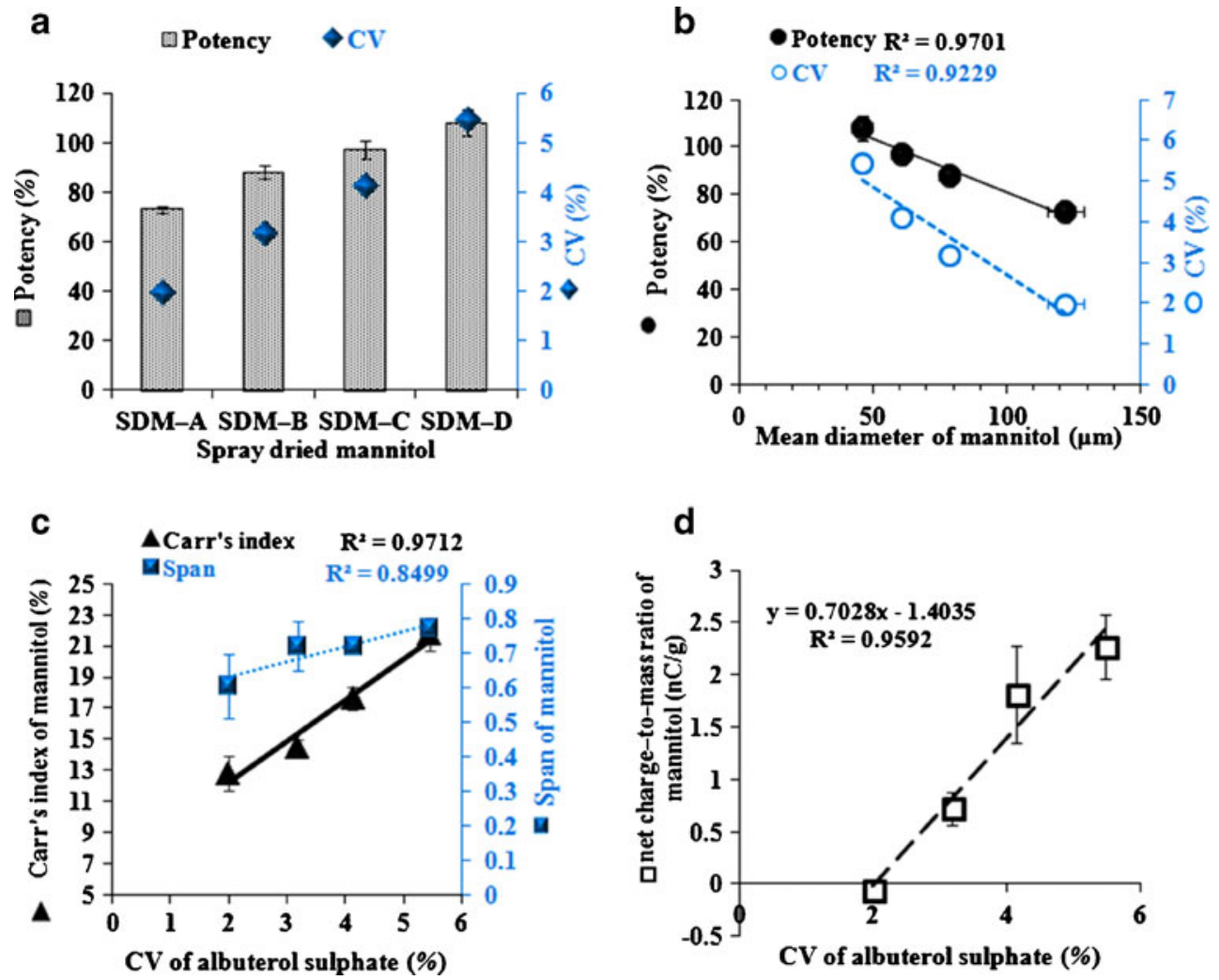

Fig. 8 Potency $(\%$, nominal dose, mean $\pm S D, n=10)$ and coefficient of variation (\% CV, $\downarrow$ ) of albuterol sulphate (AS) obtained from formulations containing AS blended separately with spray dried mannitol (SDM) particles with different size fractions: 90-125 $\mu \mathrm{m}$ (SDM-A), 63-90 $\mu \mathrm{m}$ (SDM-B), 45-63 $\mu \mathrm{m}$ (SDMC) and 20-45 $\mu \mathrm{m}$ (SDM-D) (a); potency (black circle) and \% CV (white square) of AS in relation to mean diameter (mean \pm SD, $n=10)$ of SDM (b); \% CV of AS relative to Carr's index (\% Cl, black up-pointing triangle) (mean $\pm S D, n=5)$, span $(\square)$ (mean $\pm S D, n=10)$ (c) and charge density (white square, mean $\pm S D, n=6)(\mathbf{d})$ for SDM. Homogeneity of AS drug within AS-SDM interactive mixtures is reduced in the case of SDM particles with smaller size, poorer flowability, wider size distributions and higher charge density. 
spherical shape (Fig. 2) and good-reasonable flowability (Fig. 5b, Supplementary Material 6) of all SDM particles, which could induce good drug-carrier distribution during formulation blending process. Additionally, the opposite signs of charge observed for SDM (mostly electropositive) and AS (mostly electronegative, Fig. 7e) could increase powder cohesion (and reduce powder segregation), potentially improving the physical stability of the DPI blends (37).

The following rank order can be formulated for SDM:AS formulations in terms of $\%$ potency and $\% \mathrm{CV}$ of $\mathrm{AS}$ according to SDM product: SDM-A $<$ SDM-B $<$ SDM-C $<$ SDM-D (Fig. 8a). Plotting mean diameter of SDM against $\%$ potency $\left(r^{2}=0.9701\right)$ and $\% \mathrm{CV}\left(r^{2}=0.9229\right)$ (Fig. 8b), indicating that the larger are the SDM particles the better is the drug content homogeneity. This could be attributed to the relatively more homogeneous size distribution (smaller span, Fig. 1c) and better flow properties (smaller CI, Fig.5b) of large SDM particles in comparison to smaller particles. Indeed, linear relationships were established when plotting Carr's index $\left(r^{2}=0.9712\right)$, span $\left(r^{2}=0.8499\right)$ (Fig. 8c) and net charge density $\left(r^{2}=0.9592\right)$ (Fig. 8d) of mannitol powder against \% $\mathrm{GV}$ of AS. Such relationships indicated that the variation in drug content within the DPI formulation decreased with improving the flowability, improving the size homogeneity and/or reducing the charge density of SDM carrier powder. Such findings could be explained as follow. The relatively

a

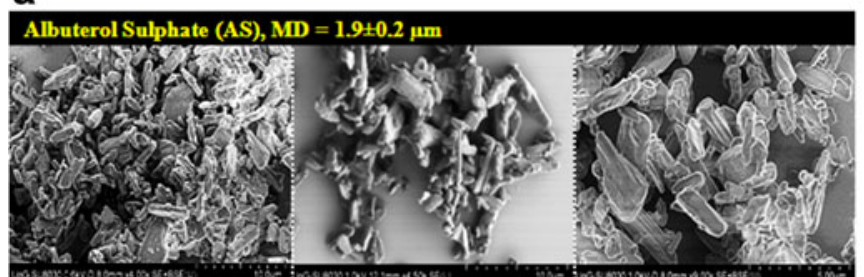

b

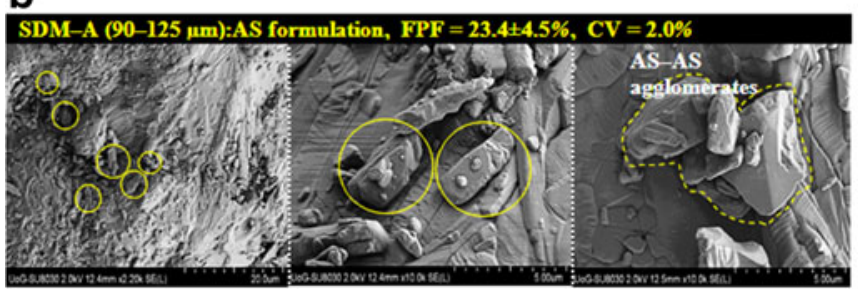

C

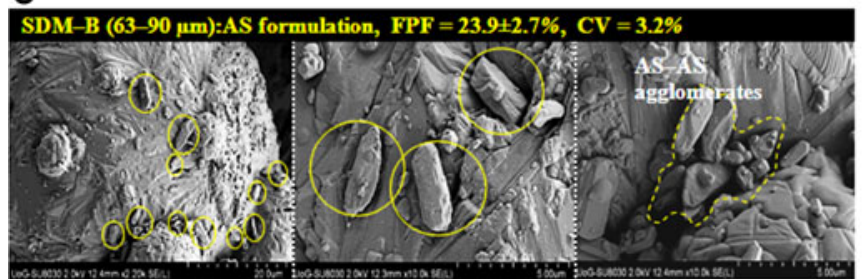

lower flowability for smaller SDM particles in comparison to larger particles could lead to poorer drug-carrier distribution during the blending process, whereas higher PSD polydispersity could enhance the creation of relatively more drug-rich areas within the formulation blends due to induced percolation segregation (isolation by size). Additionally, higher electrostatic charge density for smaller SDM particles in comparison to larger particles (Fig. 7c) could promote the formation of regions within the powder with different bulk properties promoting relatively less dose homogeneity (5). Moreover, large mannitol particles proved to have lower surface energy than smaller particles (38) potentially leading to improved drug content homogeneity because surface energy could represent an energetic barrier to drug-carrier blending (39).

Higher potency for formulations containing smaller SDM particles (Fig. 8b) could be ascribed to their relatively higher surface area (Fig. 1c), thus their ability to carry higher amounts of drug particles on their surfaces which minimize the drug 'losses' in the case of smaller SDM particles by indulging of AS in fine SDM particles.

\section{SEM/EDX Analysis for SDM:AS Formulations}

AS exhibited rectangular-shaped particles with most particles smaller than $5 \mu \mathrm{m}$ confirming their appropriateness for pulmonary drug delivery (Fig. 9a). However, a high level of
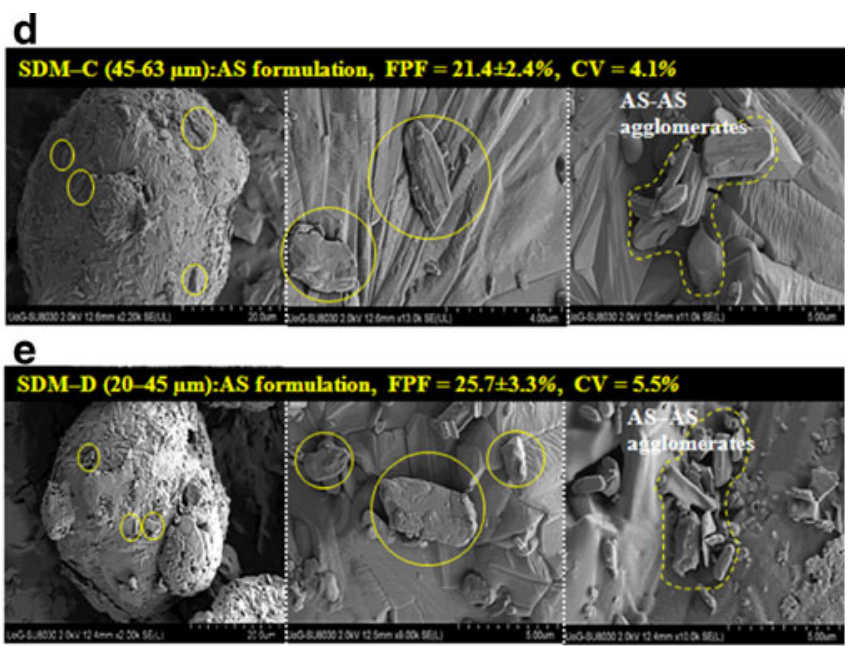

f
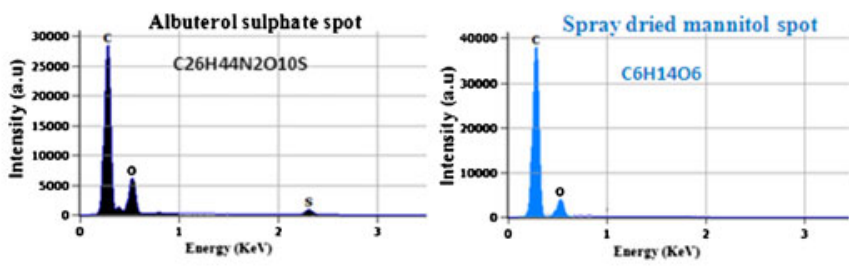

Fig. 9 SEM photographs of micronized albuterol sulphate alone (a); and formulation blends consisting of albuterol sulphate blended separately with spray dried mannitol (SDM) particles with different size fractions: 90-125 $\mu \mathrm{m}$ (SDM-A) (b), 63-90 $\mu \mathrm{m}$ (SDM-B) (c), 45-63 $\mu \mathrm{m}$ (SDM-C) (d) and 20-45 $\mu \mathrm{m}$ $(\mathrm{SDM}-\mathrm{D})(\mathbf{e})$ and scanning electron microscopy/energy-dispersive $\mathrm{X}$-ray spot analyses on albuterol sulphate-spray dried mannitol formulation mixture (f). Circles refer to AS particles. Both 'single' AS particles and AS-AS agglomerates could be detected within AS-SDM interactive mixtures. 
agglomeration was evident for AS powder, which is an intrinsic property for fine particles. Such agglomerated drug particles are likely to deposit on the upper airways upon aerosolization, thus AS powder was mixed with coarse SDM particles (Fig. 9b-e, Supplementary Material 7).

EDX in conjunction with SEM was employed to identify the presence of AS drug on the SDM carrier surface and to differentiate between AS particles and possible fine SDM particles (Fig. 9f, Supplementary Material 8). Formulations were analysed for sulphur $(S)$ element that is present as a sulphate salt of the albuterol powder but not present in mannitol (Fig. 9f). To focus on specific locations on the particle observed, spot analysis was used (Fig. 9e). Following blending with SDM particles, most AS particles were visualized as 'solo' particles (Figs. 9b-e) confirming the formation of ordered (Fig. 8a) interactive drug-carrier DPI formulation mixtures. Each interactive unit consists of small AS particles adhered to a large SDM particle as, mainly, monolayer type of adhesion (Fig. 9b-e), which was further supported by the chemical information provided by EDX analysis (Supplementary Material 8). However, some AS-AS agglomerates were present (Fig. 9a-e) as adhered to coarse SDM particles. The dispersion of AS following aerosolization is dependent on the mechanical strength of the AS-AS self-agglomerates.

Generally, a uniform distribution of elongated AS particles was observed on the spherical SDM particles suggesting a good AS content homogeneity throughout the formulations (Fig. 9b-e), which was in agreement with the homogeneity assessment of different formulations (Fig. 8a). More AS particles were adhered on 'rougher' regions of the SDM surfaces, whereas smooth regions had relatively fewer AS particles (Fig. 9b-e). AS particles located within cavities on SDM surfaces could be shielded by the mannitol surface asperities from the detachment forces generated by the turbulent airstream, thus might become entrapped and motionless during aerosolization.

\section{In Vitro Aerosolization Performance}

The amounts of drug deposited on MSLI stages 1, 2, 3, 4 and filter were not significantly influenced $(P>0.05)$ by the SDM particles with different sizes (Fig. 10a). This was also confirmed by the similar $(P>0.05)$ aerodynamic particle size distribution of AS, FPF $(21.4 \pm 2.7-25.7 \pm 3.3 \%)$, IL (59.4 $\pm 3.9-$ $64.5 \pm 6.2 \%)$, DS $(23.9 \pm 2.8-28.6 \pm 3.6 \%)$, EI $(43.8 \pm 2.3-$ $48.1 \pm 3.2 \%)$, MMAD $(2.7 \pm 0.2-2.9 \pm 0.2 \mu \mathrm{m})$ and GSD (2.1 $\pm 0.1-2.2 \pm 0.1)$ generated by all formulations (Fig. 10a). Such data indicate that SDM powders with different sizes are expected to deliver the same proportion of the dose to the different regions of the airways.

Similar MMAD (Fig. 10b) suggested a similar AS-AS degree of agglomeration within all formulations (Fig. 9b-e).
Experimental aerodynamic diameter of AS (MMAD, assessed by MSLI) (Fig. 10b) was larger than its geometric diameter $\left(d_{50 \%}=1.7 \pm 0.1 \mu \mathrm{m}\right.$, assessed by laser diffraction). This could be attributed to the presence of some AS-AS agglomerates with the formulations (Fig. 9b-e), which possibly could not be separated during aerosolization. Additionally, since AS particles demonstrated a relatively high charge density (Fig. 7e), AS particles could self-coagulate during aerosolization forming some agglomerates within the airstream (5). A GSD higher than 1.25 (Fig. 10b) confirmed that the aerosolized AS particles were broadly distributed in size.

Formulation blends with improved device emptying is considered advantageous in DPI systems (40). Amounts of AS remained in the $\mathrm{I}+\mathrm{M}$ or deposited on the throat (IP) varied significantly (Fig. 11a). Correlations were established demonstrating higher amounts of AS deposited on I+M or IP for SDM powders with higher charge density (Fig. 11b) and poorer flowability (higher CI) (Fig. 11c). This can be explained as mannitol particles with higher electrostatic charge and poorer flowability could generate more cohesive powders, thus become harder to fluidize via the airflow (may not be completely deagglomerated by the Aerolizer ${ }^{\circledR}$ device). Less cohesive powders (with lower electrostatic charge and better flowability) fluidize more equivalently by an erosion mechanism (41). In addition, particles with higher charge density could have higher probability to deposit on the inhaler device surface by electrical precipitation (5).

SDM carriers demonstrated improved aerosolization performance compared to both commercial mannitol (FPF $=14.8 \pm 1.1 \%$ (14)) and commercial lactose (FPF $=19.7 \pm 1.9 \%(42))$, suggesting SDMs as promising carriers for use in DPI systems. This could be attributed to the uniform spherical and more regular shape for spray dried mannitol particles in comparison to the micronized commercial mannitol (14) and the micronized commercial lactose (42), which could lead to reduced drug-carrier contact area and consequently better drug aerosolization performance. Unlike SDM, other important physical properties change with particle size in the case of commercial lactose such as true density, degree of crystallinity and brittleness. For example, large commercial lactose particles $(>45 \mu \mathrm{m})$ have brittle properties and delivered lower amounts of drug to the lower stages of the impactor, whereas smaller lactose particles $(<45 \mu \mathrm{m})$ have ductile properties and delivered higher amounts of drug to the lower stages of the impactor.

Although DPI formulation performance in terms of drug content homogeneity (following blending and before aerosolization) was correlated with the charge density of mannitol (Fig. 8d), the different charging behaviour for SDM powders (Fig. 7) did not significantly influence the FPF of drug (following aerosolization of AS from the Aerolizer ${ }^{\circledR}$ device) (Fig. 10b). Because aerosolization is more energetic than 

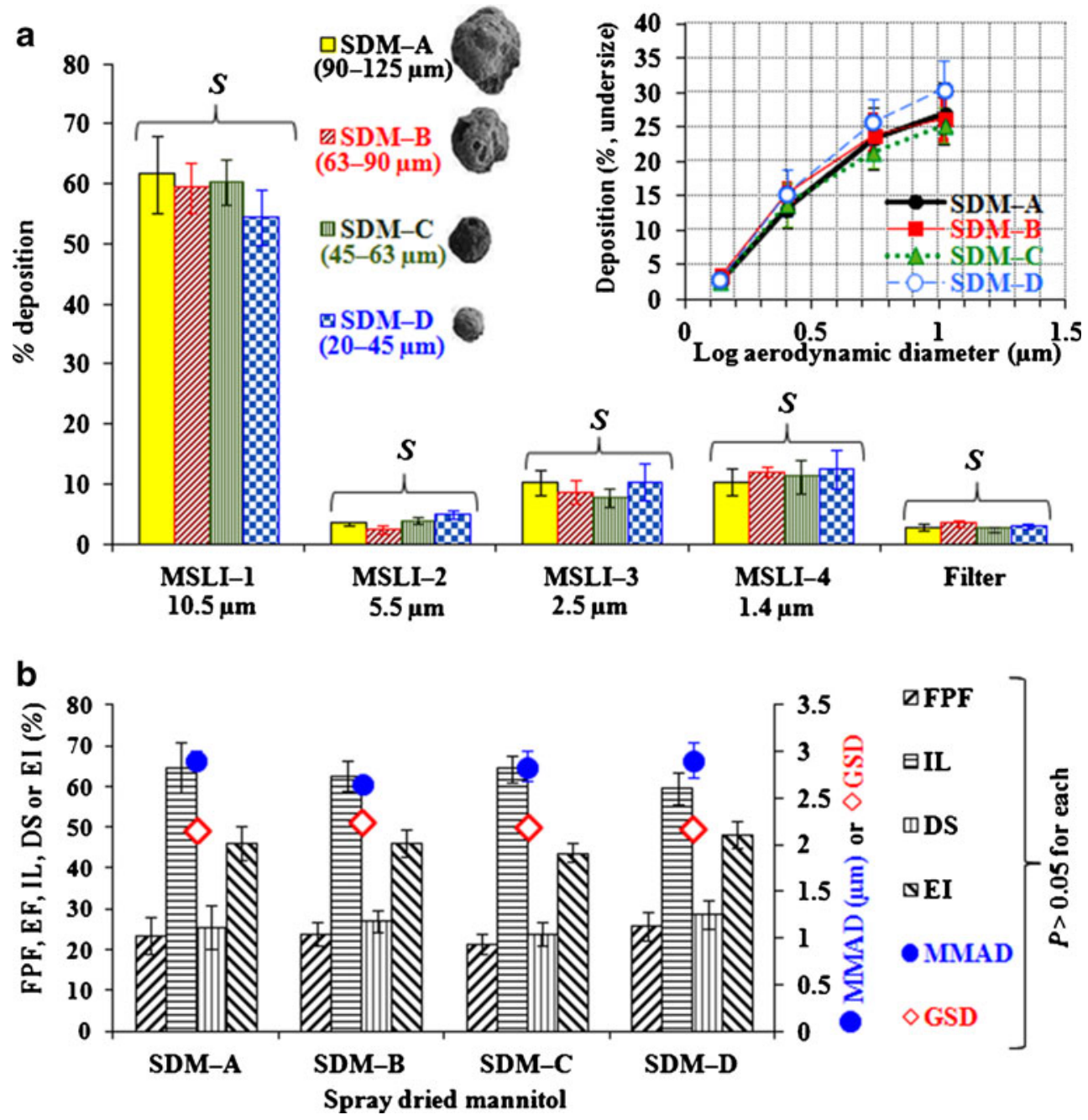

Fig. I0 Amounts of albuterol sulphate drug (AS) (\%, nominal dose) deposited on Multi-Stage Liquid Impinger (MSLI) stage I (MSLI-I), stage 2 (MSLI-2), stage 3 (MSLI-3), stage 4 (MSLI-4) and Filter; and aerodynamic particle size distribution of AS obtained from formulations containing AS blended with spray dried mannitol (SDM) particles with different size fractions: 90-125 $\mu \mathrm{m}$ (SDM-A), 63-90 $\mu \mathrm{m}$ (SDM-B), 45-63 $\mu \mathrm{m}$ (SDM-C) and 20-45 $\mu \mathrm{m}$ (SDM-D) (a); fine particle fraction (FPF), impaction loss (IL), dispersibility (DS), effective inhalation index (El), mass median aerodynamic diameter (MMAD, $\bullet$ ) and geometric standard deviation (GSD, $\diamond$ ) obtained from formulations containing AS blended with SDM particles (mean $\pm S D, n=4$ ). ' $S$ ' indicates statistically similar $(P>0.05)$. SDM particles demonstrated similar aerosolization performance.

blending, it can be assumed that the initial charges of SDM powders before aerosolization are negligible in magnitude in comparison to the charges generated during powder dispersion (35). Therefore, the original levels of charge of mannitol powders were inadequate to influence the FPF of drug following aerosolization.

The DF obtained for different formulations was in the following rank order according to SDM product: SDM-A > SDM-B $>$ SDM-C $>$ SDM-D (Fig. 12). Interestingly, regression analysis indicated that the overall DPI formulation desirability increase linearly with increasing the mean diameter of SDM particles (Fig. 12). This suggests that in the case of carrier particles with similar spherical morphology, solid-state and true density properties, larger particles outperform smaller particles within DPI formulations in terms of total desirability, at least for the model drug (AS) and model inhaler device (Aerolizer ${ }^{\circledR}$ ) used in this study.

Practically, it is nearly impossible to calculate the actual drug-carrier interaction quantitatively since many factors are involved in this process. Despite all formulations demonstrated equivalent in vitro aerosolization performance, more efforts would be required to test the lung deposition profiles in vivo and at a wider range of drugs, inhaler devices and flow rates. Indeed, it can be expected that the effect of particle size of carrier particles on DPI performance might be masked or accentuated depending on the materials used during formulating. For example, the relatively low cohesive properties of albuterol sulphate might contribute to the negligible variations in the formulations containing different spray dried mannitol particles. 

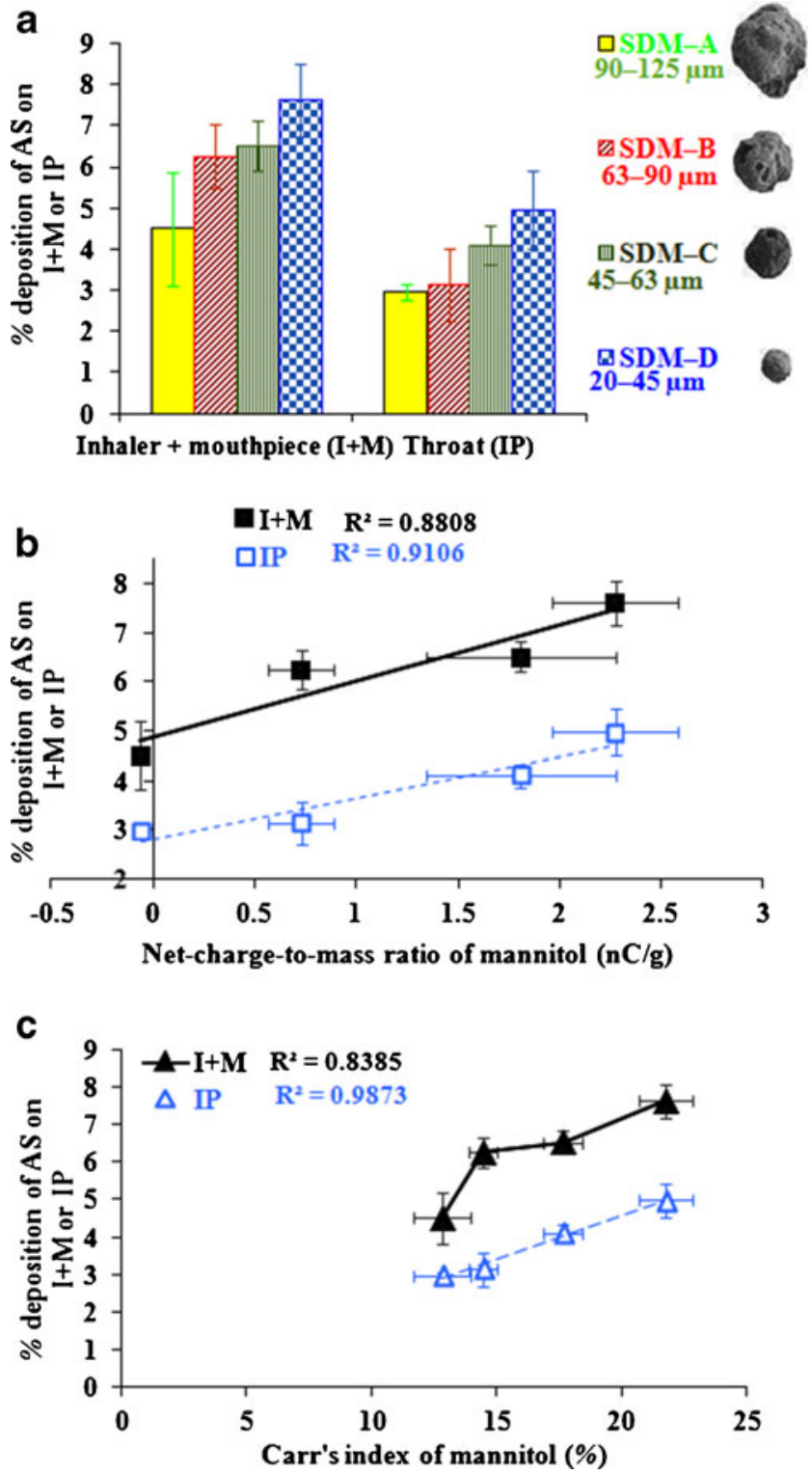

Fig. II Amounts of albuterol sulphate drug (AS) (\%, nominal dose) deposited on inhaler with mouthpiece adaptor $(I+M)$ and throat (induction port, IP) (mean \pm SD,$n=4)(\mathbf{a})$; amounts of AS deposited on I+M or IP in relation to charge density (mean $\pm \mathrm{SD}, n=6)(\mathbf{b})$ and Carr's index (mean $\pm S D, n=5)$ (c) of spray dried mannitol (SDM) obtained from formulations containing AS blended with SDM particles with different size fractions: 90 $125 \mu \mathrm{m}$ (SDM-A), 63-90 $\mu \mathrm{m}$ (SDM-B), 45-63 $\mu \mathrm{m}$ (SDM-C) and 20$45 \mu \mathrm{m}$ (SDM-D). SDM powders with higher charge density and poorer flowability deposited higher amounts of AS drug on I+M and IP.

\section{CONCLUSION AND OUTLOOK}

This study showed the effect of particle size of an attractive carrier (i.e., mannitol) that has uniform physical properties in terms of shape, solid-state form and true density on dry powder aerosol performance. Larger mannitol particles were more desirable than smaller particles due to the minimized dose variability, better flowability, reduced oropharyngeal deposition and lower charge density. Small mannitol particles produced similar fine particle fraction of drug to large particles.

Particle size had no significant effect on particle shape, true density and solid-state of spray dried mannitol particles. However, the smaller the size of the spherical mannitol particles the higher the particle surface area, the smoother the particle surface texture, the higher are the powder bulk and tap densities, the poorer the powder flowability and the higher the powder electrostatic charge of mannitol, but all the latter factors had a negligible influence on drug aerosolization behaviour. This suggested that the morphology and solid-state form of carrier particles could be determining properties in predicting the DPI aerosolization performance over carrier particle size and carrier powder bulk and charge properties.

As particle size of spray dried mannitol decreased, spray dried mannitol particles became more electro-positively charged and less electro-negatively charged. Poorer or enhanced aerosolization behaviour was not a property inherent to larger size of mannitol carrier particles. For example, in the case of 'uniform' spherical carrier particles, size is believed to be a minor factor affecting DPI performance. We believe that the controversial conclusions reported by different studies could be due to the interdependence of physicochemical properties on one another. Therefore, a great caution must be kept in mind when relating DPI performance deposition parameters with a physical property of carrier particles because it might not be the controlling factor influencing it. This research is believed to have a significant application in formulation design and processing strategies for pulmonary drug delivery systems.

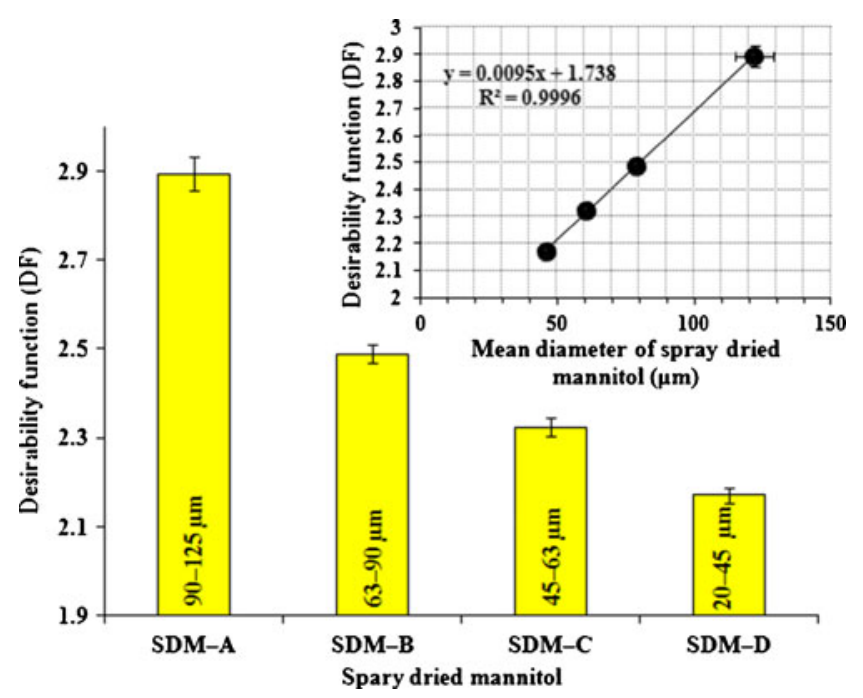

Fig. 12 Overall desirability (DF) (mean $\pm S D, n=4$ ) for formulations containing albuterol sulphate blended with spray dried mannitol (SDM) particles with different size fractions: 90-125 $\mu \mathrm{m}$ (SDM-A), 63-90 $\mu \mathrm{m}$ (SDM-B), 45-63 $\mu \mathrm{m}$ (SDM-C) and 20-45 $\mu \mathrm{m}$ (SDM-D) and DF in relation to mean diameter (mean $\pm S D, n=10$ ) of SDM. The overall desirability of the DPI formulations increases with increasing particle size of SDM. 
However, it must be kept in mind that data provided in the present study were restricted to one model of an inhaler device and one model of drug. A different inhaler device or drug (e.g., more cohesive drug) might generate different formulation performances.

\section{ACKNOWLEDGMENTS AND DISCLOSURES}

The authors thank Dr. I.J. Slipper (University of Greenwich) for help provided with SEM analyses. Waseem Kaialy and Ali Nokhodchi thank Dr. D. Tong (University of Greenwich) for access to powder charge analyses, as well as Dr. M. Maniruzzaman and Dr. D. Douroumis (University of Greenwich) for access to BET surface area analyses. Merck KGaA (Germany) is acknowledged for providing delta mannitol $\left(\right.$ Parteck ${ }^{\circledR}$ Delta M).

\section{REFERENCES}

1. Saint-Lorant G, Leterme P, Gayot A, Flament MP. Influence of carrier on the performance of dry powder inhalers. Int J Pharm. 2007;334(1-2):85-91.

2. Al-Qadi S, Grenha A, Carrión-Recio D, Seijo B, Remuñán-López C. Microencapsulated chitosan nanoparticles for pulmonary protein delivery: in vivo evaluation of insulin-loaded formulations. J Control Release. 2012;157(3):383-90.

3. Le VNP, Thi THH, Robins E, Flament M. Dry Powder Inhalers: Study of the Parameters Influencing Adhesion and Dispersion of Fluticasone Propionate. AAPS Pharm Sci Tech. 2012a:1-8.

4. Hassan MS, Lau R. Inhalation performance of pollen-shape carrier in dry powder formulation: effect of size and surface morphology. Int J Pharm. 2011;413:93-102.

5. Zeng XM, Martin GP, Marriott C. Particulate interactions in dry powder formulations for inhalation. Informa Health Care; 2001.

6. Traini D, Young PM, Thielmann F, Acharya M. The Influence of Lactose Pseudopolymorphic Form on Salbutamol Sulfate-Lactose Interactions in DPI Formulations. Drug Dev Ind Pharm. 2008;34(9):992-1001.

7. Byron PR, Jashnam R. Efficiency of aerosolization from dry powder blends of terbutaline sulfate and lactose NF with different particle-size distributions. Pharm Res. 1990;7:881.

8. Donovan MJ, Kim SH, Raman V, Smyth HD. Dry powder inhaler device influence on carrier particle performance. J Pharm Sci. 2012;101:1107.

9. Tee SK, Marriott C, Zeng XM, Martin GP. The use of different sugars as fine and coarse carriers for aerosolised salbutamol sulphate. Int J Pharm. 2000;208(1-2):111-23.

10. ShurJ, Harris H, Jones MD, Kaerger JS, Price R. The role of fines in the modification of the fluidization and dispersion mechanism within dry powder inhaler formulations. Pharm Res. 2008;25(7):1631-40.

11. Cline D, Dalby R. Predicting the quality of powders for inhalation from surface energy and area. Pharm Res. 2002;19(9):1274-7.

12. Sethuraman VV, Hickey AJ. Powder properties and their influence on dry powder inhaler delivery of an antitubercular drug. AAPS PharmSciTech. 2002;3(4):7-16.

13. Bosquillon C, Lombry C, Preat V, Vanbever R. Influence of formulation excipients and physical characteristics of inhalation dry powders on their aerosolization performance. J Control Release. 2001;70(3):329-39.
14. Kaialy W, Larhrib H, Ticehurst MD, Nokhodchi A. Influence of batch cooling crystallization on mannitol physical properties and drug dispersion from dry powder inhalers. Cryst growth Des. 2012;12:3006-17.

15. Saleem I, Smyth H, Telko M. Prediction of dry powder inhaler formulation performance from surface energetics and blending dynamics. Drug Dev Ind Pharm. 2008;34(9):1002-10.

16. Kumon M, Machida S, Suzuki M, Kusai A, Yonemochi E, Terada $\mathrm{K}$. Application and mechanism of inhalation profile improvement of DPI formulations by mechanofusion with magnesium stearate. Chem Pharm Bull. 2008;56(5):617-25.

17. Kaialy W, Alhalaweh A, Velaga SP, Nokhodchi A. Effect of carrier particle shape on dry powder inhaler performance. Int J Pharm. 2011;421:23.

18. Narayan P, Hancock B. The influence of particle size on the surface roughness of pharmaceutical excipient compacts. Mater Sci Eng, A. 2005;407(1):226-33.

19. Hüttenrauch R. Modification of starting materials to improve tabletting properties. Pharm Ind. 1983;45(4):435-40.

20. Van Campen L, Amidon G, Zografi G. Moisture sorption kinetics for water-soluble substances I: Theoretical considerations of heat transport control. J Pharm Sci. 1983;72(12):1381-8.

21. Zhu K, Tan RBH, Chen F, Ong KH, Heng PWS. Influence of particle wall adhesion on particle electrification in mixers. Int $\mathrm{J}$ Pharm. 2007;328(1):22-34.

22. Kaialy W, Martin GP, Larhrib H, Ticehurst MD, Kolosionek E, Nokhodchi A. The influence of physical properties and morphology of crystallised lactose on delivery of salbutamol sulphate from dry powder inhalers. Colloid Surface B. 2012;89:29-39.

23. Kaialy W, Momin MN, Ticehurst MD, Murphy J, Nokhodchi A. Engineered Mannitol as an alternative carrier to enhance deep lung penetration of salbutamol sulphate from dry powder inhaler. Colloid Surface B. 2010;79:345-56.

24. Kaialy W, Larhrib H, Martin GP, Nokhodchi A. The effect of engineered mannitol-lactose mixture on dry powder inhaler performance. Pharm Res. 2012;29:2139-56.

25. Mora GF, Kwan AKH. Sphericity, shape factor, and convexity measurement of coarse aggregate for concrete using digital image processing. Cem Concr Res. 2000;30(3):351-8.

26. Kaialy W, Nokhodchi A. Freeze-Dried Mannitol for Superior Pulmonary Drug Delivery via Dry Powder Inhaler. Pharm Res. 2012:1-20.

27. Kaialy W, Martin GP, Ticehurst MD, Royall P, Mohammad MA, Murphy J, et al. Characterisation and deposition studies of recrystallised lactose from binary mixtures of ethanol/butanol for improved drug delivery from dry powder inhalers. AAPS J. 2011;13:30-43.

28. Hussain T, Kaialy W, Deng T, Bradley MSA, Nokhodchi A, Armour-Chélu D. A novel sensing technique for measurement of magnitude and polarity of electrostatic charge distribution across individual particles. Int J Pharm. 2012;441:781-9.

29. Kaialy W, Ticehurst MD, Nokhodchi A. Dry powder Inhalers: mechanistic evaluation of lactose formulations containing salbutamol sulphate. Int J Pharm. 2012;423:184-94.

30. Karner S, Urbanetz NA. Triboelectric characteristics of mannitol based formulations for the application in Dry Powder Inhalers. Powder Technol. 2012:235:349-58.

31. Rowe RC, Sheskey PJ, Marian EQ. Handbook of pharmaceutical excipients. 2009: 424.

32. Das S, Larson I, Young P, Stewart P. Surface energy changes and their relationship with the dispersibility of salmeterol xinafoate powders for inhalation after storage at high RH. Eur J Pharm Sci. 2009;38(4):347-54.

33. Littringer EM, Noisternig MF, Mescher A, Schroettner H, Walzel P, Griesser UJ, Urbanetz. NA. The morphology and various densities of spray dried mannitol. Pow Technol. 2013;246:193-200. 
34. Larhrib H, Cespi M, Dyas M, Roberts M, Ford J. Engineered carrier with a long time of flight (TOF) to improve drug delivery from dry powder inhalation aerosols. Drug Delivery to the Lung (DDL). 2006;17:304-7.

35. Adi H, Kwok PCL, Crapper J, Young PM, Traini D, Chan HK. Does electrostatic charge affect powder aerosolisation? J Pharm Sci. 2010;99(5):2455-61.

36. Staniforth JN. Performance-modifying influences in dry powder inhalation systems. Aerosol science and technol. 1995;22(4):346-53.

37. Pu Y, Mazumder M, Cooney C. Effects of electrostatic charging on pharmaceutical powder blending homogeneity. J Pharm Sci. 2009;98(7):2412-21.

38. Ho R, Wilson DA, Heng JYY. Crystal habits and the variation in surface energy heterogeneity. Cryst Growth Des. 2009;9(11):4907-11.
39. Schiavone H, Palakodaty S, Glark A, York P, Tzannis ST. Evaluation of SCF-engineered particle-based lactose blends in passive dry powder inhalers. Int J Pharm. 2004;281(1-2):5566.

40. Heng D, Lee SH, Ng WK, Chan H-K, Kwek JW. Novel alternatives to reduce powder retention in the dry powderinhaler during aerosolization. Int J Pharm. 2013;452:194-200.

41. Shrimpton J. The challenge of predicting particle dynamics in dry powder inhalers.Proceedings of Drug Delivery to the Lungs 16, The Aerosol Society, London, UK, 2005, pp. 84-88.

42. Kaialy W, Ticehurst MD, Murphy J, Nokhodchi A. Improved aerosolization performance of salbutamol sulfate formulated with lactose crystallized from binary mixtures of ethanol-acetone. J Pharm Sci. 2011;100:2665-84. 